auch das unterlassene Erinnern seitens des Gläubigers als verschuldet erscheinen, und dann ist für die Anwendung des $\S 254$ Raum.

5. Diese Auffassung liegt übrigens in einem Teil ihrer Grundzüge schon einem neuern Ausspruch des Reichsgerichts ${ }^{1}$ ) zugrunde, wonach die Mahnung „ihrer Natur nach nur den Sinn und Zweck haben kann, eine geschuldete Leistung fällig zu machen und den Schuldner in Verzug zu setzen“. Da die Mahnung nach $\S 284.1$ erst nach Eintritt der Fälligkeit zulässig ist, so kann mit der Fälligkeit, die erst durch sie herbeigeführt werden soll, nur die Vollfälligkeit, mit der Mahnung nur die Kündigungs-, nicht die Erinnerungsmahnung gemeint sein. Wenn ihr aber außerdem der Zweck beigelegt wird, den Schuldner in Verzug zu setzen, so wird das nur so verstanden werden können, daß sie auch für den subjektiven Tatbestand des Verschuldens als Kriterium zu dienen hat.

\title{
III. \\ Nouvelles observations sur la date de la loi Aebutia.
}

\author{
Par \\ P. F. Girard.
}

Italiam non sponte sequor. Ce n'est pas moi qui ai voulu cet article. Celui qui l'a voulu, c'est mon excellent et honoré collègue M. le Professeur Wlassak qui, dans le dernier $n^{\circ}$ de cette Revue ${ }^{2}$ ), m'a sommé de dire si je n'abandonnais pas en face de ses objections les idées exposées par

1) Seuff. 60 S. 53. - $\left.{ }^{2}\right)$ Der Gerichtsmagistrat im gesetzlichen Spruchverfahren, Zweiter Teil, Z.S.St., 28, 1907, Rom. Abt., pp. 1-114. Erster Teil, Z.S.St., 25, 1904, Rom. Abt., pp. 81-188. Il a été fait des tirages à part dont les pages correspondent, les pp. 1-108 au premier article et les pp. 109-222 au second.

Zeitschrift für Rechtsgeschichte. XXIX. Rom. Abt. 
moi ici même en 1893.') J'obéirai à son désir en répondant que je n'abandonne rien et en disant pourquoi.

\section{I.}

Mon article de 1893 sur la date de la loi Aebutia a été inspiré par l'idée à mon avis élémentaire et alors trop méconnue ${ }^{2}$ ) que la date des monuments législatifs sur la confection desquels nous n'avons pas de témoignages directs n'est pas pour cela livrée aux fantaisies individuelles, qu'elle peut exclusivement, mais parfaitement être déterminée d'une façon plus ou moins étroite, mais sûre, à l'aide des témoignages indirects qui supposent le monument législatif existant à un certain moment, inexistant à un autre. J'ai essayé d'appliquer cette idée à la loi également importante pour l'histoire du droit et de la procédure qui se trouve mentionnée seulement dans deux textes d'Aulu-Gelle et de Gaius, dont le premier, parlant d'un certain nombre d'antiquités juridiques en désuétude, dit que ces vieilleries des Actions de la loi ont disparu à la suite de la loi Aebutia sauf dans les procès déférés aux centumvirs ${ }^{3}$ ) et le second que les Actions de la loi ont été supprimées et les formules établies entre citoyens par la loi Aebutia et deux lois Juliae. ${ }^{4}$ ) Et

1) La date de la loi Aebutia, Z.S.St., 14, 1893, Rom. Abt., pp. 11-54. - $\left.{ }^{2}\right)$ M. Wlassak écrit, Z.S.St., 28, p. 110, n. 1, que le succès de mon article a provoqué des imitations qu'il condamne également. Je n'ai pas à défendre des auteurs qu'il ne nomme pas. Mais il suffirait, pour démontrer que le travail d'assainissement entrepris là et dans mon Manuel n'a pas été inutile, de comparer, dans les ouvrages successifs de certains auteurs desquels, j'ai hâte de le dire, n'est pas M. Wlassak, les dates aventureusement précises données avant la publication de mon article et de mon livre et celles d'une approximation infiniment plus prudente données après. - ${ }^{3}$ ) 16, 10,8: Sed enim cum sproletarii « et 》adsidui« et »sanates《et »vades《 et »subvades « et »viginti quinque asses« et »taliones « » furtorumque quaestio cum lance et licio « evanuerint omnisque illa duodecim tabularum antiquitas nisi in legis actionibus centumviralium causarum lege Aebutia lata consopita sit. - 4) 4, 30: Sed istae omnes legis actiones paulatim in odium venerunt. Namque ex nimia subtilitate veterum qui tunc iura condiderunt eo res perducta est, ut vel qui minimum errasset litem perderet. Itaque per legem Aebutiam et duas Iulias sublatae sunt istae legis actiones effectumque est, ut per concepta verba id est per formulas litigemus. 
j'ai indiqué les termes extrêmes entre lesquels la loi me paraît pouvoir être enfermée. ${ }^{1}$ )

La procédure formulaire introduite d'après Gaius par la loi Aebutia me semble ne pouvoir pas encore exister vers le milieu du VI ${ }^{e}$ siècle de Rome où la troisième division des tripertita de Sex. Aelius Paetus consacrés aux XII tables, à leur interprétation et à la procédure était exclusivement relative aux legis actiones ${ }^{2}$ ), ni à la fin du même VI ${ }^{e}$ siècle où la loi Vallia prend encore la peine de remanier l'une des Actions de la loi en transformant toutes les manus iniectiones sauf deux en manus iniectiones purae ${ }^{3}$ ), ni enfin au temps de la loi Calpurnia de repetundis de l'an 605 de Rome et de la loi Junia postérieure selon lesquelles l'action en répétition des sommes extorquées aux provinciaux par les magistrats devait être intentés par la legis actio sacramenti. $\left.{ }^{4}\right)$

En sens inverse j'ai écrit que, le droit du magistrat d'empêcher l'exercice d'une action par le refus de son concours, les exceptions, les actions prétoriennes et même les

1) Je suis parti et je pars toujours de la conception de la portée de la loi Aebutia due à $\mathrm{M}$. Wlassak, à savoir que cette loi, après laquelle on trouve encore toutes les Actions de la loi et déjà partout les formules, a donné le choix entre les deux procédures aux parties sous le contrôle du magistrat. Mais mon argumentation s'adapte aussi bien au système de M. Bekker, Z.R.G., 5, 1866, pp. 341-356, qui admet pareillement la coexistence des Actions de la loi et des formules en supposant que la loi Aebutia aurait pour chaque procès juxtaposé la formule à la legis actio. Elle s'appliquerait moins bien aux systèmes selon lesquels la substitution des formules aux legis actiones aurait été faite pour certaines Actions de la loi par la loi Aebutia et pour les autres par les lois Juliae, notamment au seul d'entre eux qui soit encore soutenu, à celui de M. Eisele, Abhandlungen z. römisch. Civilproceß, 1889, pp. 67-109, et de M. Cuq, Institutions juridiques des Romains, $1^{2}$, 1904, p. 287, selon lequel la loi Aebutia n'aurait introduit la formule et supprimé la legis actio qu'en matière de condictio. Mais précisément les faits que nous invoquons sont des arguments contre cette doctrine. - $\left.{ }^{2}\right)$ Pomponius, D., 1, 2, 2, 38: . . exstat illius liber qui inscribitur tripertita, qui liber veluti cunabula iuris continet: tripertita autem dicitur, quoniam lege duodecim praeposita iungitur interpretatio, deinde subtexitur legis actio. - ${ }^{3}$ ) Gaius, 4, 25. - 4) Loi Acilia, ligne 23: [Qwod cum eo lege Calpu]rnia aut lege Iunia sacramento actum siet. Cf. lignes 74. 81. 
actions de bonne foi n'ayant existé que depuis l'introduction de la procédure formulaire, la loi Aebutia était forcément antérieure à la première apparition de tout cela: du droit de denegare legis actionem attesté par un récit de Cicéron en 627 ou $628^{1}$ ); de l'action de mandat donnée comme action de bonne foi ou plus probablement comme action prétorienne in factum, par Sex. Julius préteur urbain en $631^{2}$ ); de la formule rutilienne du bonorum emptor créée au plus tard en 636 par le préteur P. Rutilius Rufus ${ }^{3}$ ); de l'édit du même préteur Rutilius restreignant l'effet légal du iudicium operarum ${ }^{4}$ ); de l'action de mandat, sans doute in ius et de bonne foi, accordée contre les héritiers, entre l'an 637 et l'an 639 par le préteur M.Livius Drusus ${ }^{5}$ ); de l'édit d'un préteur Drusus qui est sans doute le même refusant la vindicatio in libertatem à l'affranchi qui ne veut pas renouveler après l'affranchissement le serment relatif aux operae ${ }^{6}$ ); des mentions faites en 643 par la loi agraire de la venditio bonorum et de tout un groupe d'exceptions ou de prescriptions ${ }^{7}$ );

1) De orat., 1, 36, 166: Potes igitur, inquit Crassus, ... oratores putare eos, quos multas horas exspectavit, cum in campum properaret, et ridens et stomachans Scaevola, cum Hypsaeus (cos. 629) maxima voce, plurimis verbis a $M$. Crasso praetore contenderet, ut ei, quem defendebat causa cadere liceret, $C$ n. autem Octavius homo consularis (cos. 626) non minus longa oratione recusaret, ne adversarius causa caderet ac ne is, pro quo ipse diceret, turpi tutelae indicio atque omni molestia stultitia adversarii liberaretur? $167 \ldots$ alter plus lege agendo petebat, quam quantum lex in XII tabulis permiserat; quod cum impetrasset, causa caderet: alter iniquum putabat plus secum agi, quam quod erat in actione, neque intelligebat, si ita esset actum, litem adversarium perditurum. - ${ }^{2}$ ) Ad Her., 2, 13, 19: M. Drusus, praeter urbanus (tr. pl. 632, cos. 642), quod cum herede mandati ageretur, iudicium reddidit, Sex. Iulius (pr. urb. 631, De domo, 53) non reddidit. - ${ }^{3}$ ) Gaius, 4, 35: Quae species actionis appellatur Rutiliana, quia a praetore $P$. Rutilio (cos. 649, mais déjà candidat au consulat pour 639: Cicéron, Brut., 30,113), qui et bonorum venditionem introduxisse dicitur, comparata est. - $\left.{ }^{4}\right)$ D., 38, 2, 1, 1: Et quidem primus praetor Rutilius edixit se amplius non daturum patrono quam operarum et societatis actionem, videlicet, si hoc pepigisset, ut nisi et obsequium praestaret libertus in societatem admitteretur patronus. s) Ad Her., 2, 13,19 (n. 2). - " ) Cicéron, Ad Att., 7, 2, 8: Itaque usurpavi vetus illud Drusi, ut ferunt, praetoris in eo, qui eadem liber non iuraret, me istos liberos non addixisse, praesertim cum adesset nemo, a quo recte vindicarentur. - ${ }^{7}$ ) Ligne 56: [U]tei curator eius 
des actions de bonne foi énumérées encore un peu plus tard par Q. Mucius Scaevola, le consul de $659^{1}$ ) et, par exemple de l'édit refusant la vindicatio in libertatem à l'homme libre qui s'est laissé vendre pour partager le prix auquel le même Q. Mucius fait allusion ${ }^{2}$ ), comme de tous les évènements plus récents qui supposent chez le magistrat une autorité qu'il a eue depuis la loi Aebutia et qu'il n'avait pas auparavant. Et j'estime avoir ainsi localisé la loi Aebutia dans le premier tiers du VII ${ }^{\theta}$ siècle, un peu après l'an 605 de Rome $=149$ av. J.-C., un peu avant l'an $628=126$.

Depuis j'ai reproduit mon argumentation en tout ou en partie, soit dans mon Manuel élémentaire de droit romain ${ }^{3}$ ), soit dans une réimpression de l'article de cette Revue publiée en 1897 par la Nouvelle Revue historique de Droit ${ }^{4}$ ), soit dans le tome I de mon ouvrage sur l'Organisation iudiciaire des Romains ${ }^{5}$ ), en n'en changeant naturellement pas les termes essentiels, en y faisant seulement quelques légères additions, ainsi en signalant dans la réimpression de l'article et dans les dernières éditions de mon Manuel la preuve de l'existence de la formule au début du $\mathrm{VI}^{e}$ siècle relevée par M. Kniep dans la loi latine de Bantia ${ }^{6}$ ), ainsi, dans le

profiteatur, item ute[i ... ex e]o edicto, utei is, quei ab bonorum emptore magistro curato/reve emerit]. Ligne 38: [De ea re iudicare iubeto,] quae res soluta $n$ [on siet inve iudici]o non siet iudicatave non siet, quod eius praevaricationus [causa ... vel per dolum malum petitorum patronoru] mve factum non siet.

1) Cicéron, De off., 3, 17, 70: Q. quidem Scaevola pontifex maximus summam vim esse dicebat in omnibus iis arbitriis, in quibus adderetur »ex fide bona « fideique bonae nomen existimabat manare latissime idque cersari in tutelis societatibus fiduciis mandatis rebus entis venditis conductis locatis quibus vitae societas contineretur; in iis magni esse iudicis statuere, praesertim cum in plerisque essent iudicia contraria, quid quemque cuique praestare oporteret. - 2) Paul., D., 40, 12, 23, pr. - 3) 1ère éd., 1896, p. 966, n. 2; 2 éd., 1898, p. 971, n. 2; $3^{\ominus}$ éd., 1901, p. 987, n. 2 ; $4^{e}$ éd., 1906 , p. 993 , n. 2 ; trad. allemande publiée sous le titre $G e$ schichte und System des römischen Rechts, übersetzt von R. von Mayr, 1908, p. 1090, n. 1. - 4) 21, 1897, pp. $249-296$ - s) 1, 1901, p. 195 et ss. - ${ }^{6}$ ) Lignes 9. 10: Pr(aetor) recuperatores ... dato, iubetoque eum, sei ita pariat, condumnari populo. La décision de P. Mucius Scaevola sur la dot de la veuve de C. Gracchus $(D ., 24,3,66, p r$.) serait une autre preuve de l'existence de la loi Aebutia en 633, si elle se 
volume de l'Organisation judiciaire, en soulignant le témoignage d'une muette éloquence fourni par les leges imperfectae et minus quam perfectae du $\mathrm{VI}^{\mathrm{e}}$ siècle en faveur de l'impuissance du magistrat du temps des Actions de la loi à arrêter l'exercice des actions légales. J'aurais peut-être encore, à l'occasion, indiqué quelques autres arguments complémentaires dont je me suis avisé depuis, par exemple la concomitance singulière qu'on peut observer entre l'époque où surgissent les premiers vestiges des institutions de la procédure formulaire incompatibles avec la domination exclusive des legis actiones et celle où l'on rencontre pour la première fois des procès entre citoyens déférés aux récupérateurs qui ne statuaient anciennement que sur les procès où figuraient des pérégrins. Mais je n'aurais naturellement pas écrit un nouvel article exprès pour dire cela. Au contraire, puisque je peux malaisément me refuser à une discussion sur laquelle M. Wlassak a la galanterie de se dire obligé d'insister par le succès de mes idées et qu'il me parait souhaitable d'essayer de l'élever au dessus du niveau d'un dialogue purement polémique entre lui et moi, je profiterai de l'occasion pour soumettre la question à une révision d'ensemble, en me demandant en conscience s'il existe des raisons d'abandonner ou de modifier le sentiment que j'ai exprimé à la fin de 1893 ; en examinant non seulement les contestations qui m'ont été adressées par M. Wlassak, mais celles qui me sont venues d'autres auteurs que je n'ai pas convaincus davantage, ou même sur des points particuliers d'auteurs qui acceptent ma solution du problème; en ne cherchant pas seulement quelles critiques mon système a rencontrées dans la doctrine, mais dans quelle mesure il peut se trouver contredit ou rectifië par des documents nouveaux.

rapportait sûrement, comme l'a pensé Pernice Z.S.St., 20, 1899, p.131, n. 5, à l'action rei uxoriae que M. Esmein a démontré N.R.H., 1893, pp. 145-171, être une action prétorienne in aequum et bonum concepta. Mais j'estime toujours que le texte n'est pas tout à fait probant parce qu'il pourrait s'entendre de l'action ex stipulatu (Z.S.St., 14, 1893, p. 32, n. 3, arec l'addition N.R.H., 1897, p. 272, n 3). 
II.

Sur le dernier point, un mot suffira. Il ny a pas en réalité de document nouveau. La riche collection de matériaux juridiques fournis depuis quinze ans par les papyrus égyptiens appartient tout entière au cercle de la procédure extraordinaire; elle ne nous apprend rien sur la substitution des formules aux Actions de la loi. Il en est de même des quelques autres textes ou monuments isolés de l'époque impériale, (acte d'affranchissement régi par la loi Junia, fragments d'Ulpien de Strasbourg, fragment d'Oxford de societate) découverts dans les dernières années. La loi municipale de Tarente, qui est au contraire du temps de la République, mais d'une époque où nous estimons qu'on a déjà suffisamment d'autres preuves du sysțème formulaire, n'en apporte pas de nouvelle.

Les seuls monuments, récemment découverts ou récemment produits que l'on pourrait songer à mêler au débat, seraient les inscriptions grecques relatives à des procès entre cités organisés par le Sénat romain à la fin du $\mathrm{VI}^{\mathrm{e}}$ siècle et au début du VII e, où $M$. Partsch a eu la perspicacité de relever des vestiges de la formule. ${ }^{1}$ )

$\mathrm{Si}$ ces monuments ont la portée que leur assigne $\mathrm{M}$. Partsch, ce sont des documents d'un intérêt incontestable pour l'histoire de la procédure formulaire entre pérégrins dans les provinces, peut-être en partie par contrecoup pour celle de la procédure formulaire entre pérégrins à Rome. Mais ils n'atteignent en rien les conclusions que j'ai proposées sur la date de l'introduction de la procédure formulaire à Rome entre les citoyens; car, si je suis loin de croire que les Actions de la loi aient été fermées aux non-citoyens avec l'inflexible rigueur que veut M. Wlassak ${ }^{2}$ ) et qui lui parait,

1) Die Schriftformel im römischen Provinzialprozesse, Breslau, 1905, pp. 3-52, sur Dittenberger, Sylloge inscriptionum Graecarum, $2^{\ominus}$ éd., 1900, I, 314. II, 928. 929. Cf. sur la seconde inscription nos observations, Mélanges Gérardin, 1907, p. 256, n. 1. V. aussi H. F. Hitzig, Z.S.St., 28, 1907, pp. 251-252. - $\left.{ }^{2}\right) \nabla$. en dernier lieu l'article sur l'exclusion des Latins de la legis actio, Z.S.St., 28, 1907, pp.114-129, et dans le même sens Mitteis, Römisches Privatrecht bis auf Diocletian, 1, 1908, p. 124, n. 45. J'ai indiqué la littérature plus ancienne, Organisation 
judiciaire, 1, p.213, n.4. A mon avis, le texte de Cicéron, Pro Caecina, 33, 97 et ss., sur la femme d'Arretium prouve, à la vérité au sujet de pérégrins de la pire condition, qu'il y a toujours eu des pérégrins qui, tout en ayant des droits reconnus par l'Etat romain, ne pouvaient pas les faire valoir par legis actio; mais il ne prouve pas qu'aucun pérégrin n'ait jamais pu agir par legis actio. Rationnellement la legis actio a pu être accordée ou à certains pérégrins pour tous les droits qui leur étaient reconnus ou à tous les pérégrins pour certains droits, et en fait nous avons des exemples des deux modes de concession. Pour le second, l'exemple classique nié par M. Wlassak, mais reconnu par M. Mitteis est la concession de la legis actio sacramenti faite par la loi Calpurnia de 605 aux provinciaux dépouillés par les magistrats romains (p.115, n.4). Pour le premier, je serais toujours porté à croire que les traités qui accordaient à des pérégrins donnés tout ou partie des droits civils, commercium ou même conubium, leur accordaient, dans la même mesure, le droit de les faire valoir dans la procédure civile de la legis actio; mais, en tout cas, ce me parait toujours, malgré la contestation de M. Karlowa recueillie par M. Wlassak, l'interprétation la plus exacte du traité avec Carthage de l'an 448, duquel Polybe dit, $3,24,12.13$, qu'il donnait, en matière commerciale, les mêmes droits aux Romains qu'aux Carthaginois à Carthage et aux Carthaginois qu'aux Romains à Rome; car l'égalité n'existerait pas si elle s'appliquait seulement à l'acte d'acheter ou de vendre et non à sa sanction. J'hésite encore moins à penser, contrairement à M. Wlassak et à M. Mitteis, que les Latins peuvent faire valoir à Rome par voie de legis actio tous les droits qui leur y sont reconnus, c'est-à-dire les droits qui résultaient pour tous de la concession du commercium et au moins pour certains, sans doute anciennement pour tous, de celle du conubium; car, à côté des raisons générales, il y a ici des raisons spéciales résultant de l'identité des institutions primitives de Rome et des cités latines, d'ume part, et, d'autre part, de l'alliance de Rome et des eités latines. Chaque membre de chaque cité peut, en conséquence, procéder, dans les autres cités, Rome comprise, non-seulement aux actes de la vie juridique extrajudiciaire, mais aux actes de la vie judiciaire et même aux actes de la vie politique. Le premier point est, en nous en tenant au commercium, prouvé par le texte d'Ulpien, 19, 4, qui montre que le Latin, même le Latin junien, peut aussi bien qu'un citoyen, figurer dans la mancipation, acte propre aux citoyens, d'après Gaius, 1,$119 ; 2,65$. Le troisième l'est par les textes qui montrent qu'au temps de Cicéron, on tirait encore au sort dans les comices la section de vote des Latins avant d'ouvrir le vote (v. les textes dans Mommsen, Römisches Staatsrecht, 3, p. 397, n. $1=$ tr. fr., 6, 1, p. 457, n. 1). Le second n'est pas plus surprenant: il est impliqué par les deux autres. M. Wlassak, qui ne parle pas du troisième, s'est si bien aperçu du lien qui existe entre le premier et le second qu'il a soutenu que le Latin ne peut pas plus se dire propriétaire selon le 
visiblement indispensable à l'admission intégrale de sa notion des iudicia legitima ${ }^{1}$ ), j'ai toujours considéré comme possible

droit des Quirites dans l'acte per aes et libram que dans la legis actio, qu'il $\mathrm{y}$ avait deux mancipations différentes: celle propre aux citoyens et celle servant aux rapports des citoyens et des Latins. Mais cette idée dont I'invraisemblance a fait reculer M. Mitteis (p.118, n. 20; cf. p. 120 , n. 28) est directement réfutée par Ulpien, 20, 8, qui, en disant qu'un Latin Junien peut être témoin dans la mancipation constituée par le testament per aes et libram, parle certainement de la mancipation romaine et non de la mancipation internationale imaginée par M. Wlassak. Cela réfute même par contrecoup l'argument tiré par lui du texte de Gaius 2, 65, qui présente l'in iure cessio - en même temps que la mancipation - comme propre aux citoyens. Gaius la dit propre aux citoyens au même sens que la mancipation, en négligeant les Latins et les pérégrins gratifiés du commercium qui sont indiqués dans le passage plus explicite d'Ulpien, 19, 4. Le Latin peut, comme le citoyen, dire: Aio hanc rem meam esse ex iure quiritium soit devant les témoins de l'acte per aes et libram, soit devant le magistrat de la legis actio simulée constituée par l'in iure cessio, soit devant le magistrat de la legis actio sérieuse. Et cette solution, qui ne nous choquerait pas même pour le pérégrin ordinaire pourvu du commercium, est particulièrement peu surprenante pour les Latins, qui peuvent comparaître officiellenent devant le magistrat, même quand il tient les comices, pour exprimer un vote. La communauté de participation aux comices, sur laquelle $M$. Wlassak a omis de s'expliquer, constitue un argument a fortiori pour la communauté de participation à la legis actio. J'ajoute enfin que l'admission des Latins à la legis actio est confirmée par une autre particularité signalée par Mommsen, Staatsrecht, 3, p. $603=$ tr. fr., 6, 2, p. 221. Cf. Girard, Org. Jud., 1, pp. 211-212: l'attribution de leurs procès au préteur urbain et non au préteur pérégrin dans le titre officiel duquel ils n'auraient pas été omis s'ils avaient été ses justiciables. Même parmi les procès déférés au préteur des étrangers, il y en avait, à notre avis, qui se liaient par legis actio; mais, si, à la différence des procès des citoyens, ceux des Latins n'avaient pu se lier par legis actio, ils auraient été déférés au préteur des étrangers et non à celui des citoyens.

1) A la différence de plusieurs bons jurisconsultes, je considère, pour ma part, comme l'explication la plus ingénieuse qui ait été proposée du nom des iudicia legitima, celle donnée par M. Wlassak, selon laquelle ces procès ne sont pas ceux qui ont été visés par une loi quelconque, mais ceux qui ont été visés par la loi Aebutia, ceux pour lesquels la loi Aebutia a autorisé la substitution de la formule à la legis actio, en sorte qu'il faut admettre que la loi n'a visé que les procès liés à Rome, entre citoyens, pour être déférés à un index unus. Mais M. Wlassak sera le premier à reconnaitre, malgré ce qu'il écrit, 
que la formule ait dès avant la loi Aebutia joué un certain rôle soit dans les procès où figuraient des pérégrins, soit dans les procès provinciaux. ${ }^{1}$ ) La question de l'introduction des formules dans la justice pérégrine ou provinciale et celle de leur introduction dans la justice de Rome entre citoyens sont deux questions distinctes, deux questions si distinctes que M. Partsch a pu adopter mon système sur la date où les formules furent introduites à Rome entre citoyens par la loi Aebutia, à la première page du travail où il soutient qu'elles existaient antérieurement dans les provinces. ${ }^{2}$ )

III.

En omettant M. Moritz Voigt, qui, dans son Ius naturale, avait placé la loi Aebutia entre l'an 513 et l'an 517 à l'aide de méthodes aujourd'hui abandonnées ${ }^{3}$ ) et qui, dans sa Römische Rechtsgeschichte, m'a combattu par des arguments

Z.S.St., 28, pp. $117-118$, que cela ne me force pas à abdiquer ma liberté d'examen pour adopter aveuglément toutes les solutions qui s'accorderaient le mieux ou qui lui sembleraient s'accorder le mieux avec sa doctrine. Ainsi, par ex., je ne peux, pour mieux expliquer que la loi n'ait pas visé les procès pérégrins, fermer les yeux sur les témoignages qui me paraissent prouver que les pérégrins ont agi par legis actio. Ce n'est d'ailleurs pas la seule lacune qu'il faille, avec l'explication de M. Wlassak, constater, à notre avis, dans la loi. S'il est surprenant, au point de vue des personnes, qu'elle n'ait pas visé les procès des pérégrins, il ne l'est pas moins, au point de vue des lieux, qu'elle n'ait pas visé les procès qui étaient liés entre citoyens dans les préfectures créées depuis le Ve siècle (Mommsen, Staatsrecht, 3, $580-582=$ tr. fr. 6, 2, 194-197; Girard, Org. jud., 1, pp. 295-305), donc à une date antérieure à celle où M. Wlassak place la loi Aebutia qu'il croit postérieure à Sex. Aelius, et qui devaient se lier par legis uctio devant les délégués du préteur comme devant lui. Au reste, les deux imperfections ne dépassent pas le niveau de celles qu'on rencontre dans les lois de tous les pays.

1) Z.S.St., p. $14=$ = R.R.H., p. 252. Org. jud., 1, p. 216, n. 2. 326,

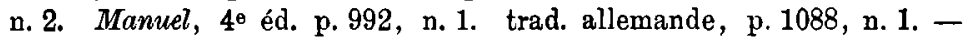
2) Schriftformel, p. 1, n. 3. C'est par conséquent, me semble-t-il, par une méprise que M. Bekker qui m'avait fait l'honneur d'adhérer à ma démonstration, Z.S.St., 15, 1894, p. 164, l'a depuis présentée incidemment, Z.S.St., 27, 1906, p. 39, comme écartée par le travail de M. Partsch. - ${ }^{3}$ ) Ius naturale, 3, 1875, p. 814 et ss. Römische Rechtsgeschichte, 1, 1892, p. 828 et ss. 
dont la discussion détaillée aurait, me semble-t-il, un peu l'air d'un anachronisme ${ }^{1}$ ), trois auteurs ont, ̀̀ ma connaissance entrepris la réfutation en forme de mes conclusions. Ce sont: M. Cuq, selon lequel la loi Aebutia dont la portée se serait réduite à la substitution de la formule à la legis actio en matière de condictio, serait au plus tard du VI ${ }^{\mathrm{o}}$ siècle $^{2}$ ); M. Wlassak, qui la met entre le milieu du VI' siècle, à cause de Sex. Aelius, et la seconde moitié du VII ${ }^{\ominus}$, ̀ cause de passages du Pro Flacco, qui est de 685, et peutêtre du Pro Roscio comoedo qu'il place entre 677 et $686^{3}$ ); enfin M. Mitteis, qui considère que le seul terme extrême absolument sûr est celui fourni par Sex. Aelius, mais qui estime probable que la loi appartient à la fin du VI siècle ou au début du VIIe, qui aboutit donc, bien que par des voies très différentes, à une solution assez voisine de la

1) Römische Rechtsgeschichte, 2, 1899, p. 420, n. 54. M. Voigt, qui ne parait pas avoir toujours parfaitement compris ce que nous écrivions (par ex. au sujet des actions édiliciennes relatives à la vente et des interdits), ne nous oppose pas seulement, comme preuve de l'existence de la loi arant la fin du VIo siècle, les textes connus de Plaute et de Sex. Aelius sur la vente que nous retrouverons invoqués dans un autre sens (p. 155 et ss.), ni comme objection à son classement au VII e siècle l'argument que nous retrouverons également (p. 154, n. 1), tiré du trop peu d'espace que cela laisserait entre elle et Cicéron. Il nous reproche notamment de n'avoir pas vu que l'existence de la loi Aebutia était établie par les preuves qui se rencontreraient de la restitutio in integrum ob absentiam dans Térence; des exceptions dans la loi Cincia et la loi Plaetoria (de laquelle en revanche il nie l'action malgré la loi Julia de 709 et le fragment de formula Fabiana); de la prorogatio fori en matière de vente dans Caton, De r. r., 149 (où il ajoute à iudicium les mots sc. ex empto vendito); du vadimonium de citation dans des passages de Tite-Live, de Valère Maxime et de Plaute relatifs aux années ${ }^{\bullet} 539,543-544$ et au début de la seconde moitié du VIe siècle; enfin ailleurs des actions confessoire et négatoire relatives à l'usufiuit et aux servitudes urbaines. - ${ }^{2}$ ) Dictionnaire des Antiquités de Daremberg et Saglio, vo Leges publicae, tome 5, p. 1127. Institutions des Romains, 2, 1902, p. 732; 1,2e éd., 1904, p. 286. L'auteur avait lui-même auparavant placé la loi dans la seconde moitié du VI0 siècle, Institutions, 1, 1891, p. 716. - ${ }^{3}$ ) La date est indiquée par M. Wlassak, Z.S.St., 28, p. 110, n. 2. M. Wlassak se prononce contre mon système nommément Z.S.St., 25, p. 131, n. 2 et Z.S.St., 28, p. 110, n. 2, mais encore en beaucoup d'autres passages, par ex. Z.S.St., 25, pp. 81, ๖. 1. 136. Z.S.St., 28, pp. 91, n. 1. 96. 98, n. 2. 105-112. 
mienne. $\left.{ }^{1}\right)$ Mais certains points de mon argumentation ont été contestés par des auteurs qui n'ont pas pris parti sur le système ou même qui ont paru l'adopter, ainsi l'argument relatif à la loi Vallia par $\mathbf{M}$. Appleton ${ }^{2}$ ), celui tiré du procès de 627-628 par M. Huvelin ${ }^{3}$ ), celui relatif au droit de denegare legis actionem par plusieurs autres. Je tâcherai de m'expliquer sur tous les points en reprenant successivement les arguments que j'ai invoqués pour l'existence et l'inexistence de la loi.

I. Parmi les trois phénomènes que j'ai relevés comme supposant l'existence de la loi, le premier et le plus ancien, qui est la mention exclusive des legis actiones dans les tripertita de Sex. Aelius, peut, je crois, être tenu pour incontesté. M. Cuq n'en dit rien, M. Mitteis et M. Wlassak l'admettent tous deux expressément.

Pour la loi Vallia que je plaçais vers la fin du VI ${ }^{e}$ siècle, parce qu'elle est postérieure à la loi Cincia de $550^{4}$ ) et qu'elle me parait devoir être antérieure à la loi Aebutia, M. Appleton a fait remarquer que, puisque les legis actiones n'ont pas été abolies par la loi Aebutia et qu'en particulier la manus iniectio subsiste encore au temps de César, la loi Vallia peut n'avoir modifié le régime des manus iniectiones qu'après la loi Aebutia. Matériellement, c'est possible. Historiquement ce ne l'est guère; car, si cette loi se comprend très bien à l'époque de la domination exclusive des Actions de la loi où son résultat ne pouvait être obtenu que par l'intervention du législateur, elle se comprendrait beaucoup moins après l'introduction de la procédure formulaire, quand il eut pu être atteint plus simplement par le préteur ${ }^{5}$ );

1) Römisches Privatrecht bis auf Diocletian, 1, 1908, p. 52, n. 30. 2) Z.S.St., 26, 1905, pp. 6-9. Mélanges Gérardin, 1907, pp. 17-18. 3) Mélanges Gérardin, 1907, p. 325, n. 2. - 4) V. p. 166, n. 2. -

s) Voir en ce sens Levy, Sponsio, fudepromissio, fideiussio, 1907, p. 61. M. Appleton, Mélanges Gérardin, p. 17, »avoue ne pas voir du tout comment au VIIe siècle, dans l'enfance de la procédure formulaire, le préteur qui a attendu jusqu'aux dernières années de ce siècle avant d'oser seulement introduire l'exception de dol aurait eu l'audace de violer ouvertement les lois nombreuses organisant dans des cas multiples la manus iniectio pro iudicato «. On peut répondre que l'enfant grandit assez vite, que le préteur eut, dès le VIIe siècle, l'audace 
c'est pour cela que les lois relatives au droit privé sont si rares depuis la loi Aebutia. ${ }^{1}$ ) Au reste, si ce point a une importance sérieuse pour d'autres questions, M. Appleton remarque avec raison qu'il en a relativement peu pour celle que nous traitons ici. ${ }^{2}$ )

Un point plus important pour nous et de beaucoup, c'est le terme extrême le plus récent, celui tiré de l'emploi exclusif de la legis actio sacramenti en matière de repetundae, en l'an 605, date de la loi Calpurnia, et à la date postérieure de la loi Junia. Après la loi Aebutia, les lois Calpurnia et Junia auraient parlé ou de formule et de legis actio ou de formule toute seule. Elles ne l'auraient pas fait de legis actio toute seule. Si elles donnent la legis actio sacramenti comme action en répétition à leurs étrangers détroussés par les magistrats romains, c'est qu'elles ne pouvaient pas leur donner autre chose. Le témoignage est même d'autant plus frappant que l'on considère la legis actio comme une voie de droit moins appropriée aux procès des pérégrins, et cela devrait le rendre plus probant pour M. Wlassak que pour personne. Cependant M. Wlassak n'en dit rien à propos de notre question, et s'il en avait parlé, ģ'aurait sans doute été, comme il a fait à un autre propos, pour refuser d'en tenir compte en le taxant d'obscurité. ${ }^{3}$ ) Mais les textes précis ne s'éliminent pas par omission et celui-ci porte clairement que d'après les lois Calpurnia et Junia les pérégrins dépouil-

de violer la loi des XII tables elle-même eu refusant la rindicatio in libertatem à l'homme qui s'était laissé vendre pour partager le prix ou à l'affranchi qui refusait de répéter le serment relatif aux operae, et que ce sont précisément les lois qui paraissaient les plus odieuses contre lesquelles il a dû le plus facilement oser intervenir.

1) V. les pp. 166 et ss. - $\left.{ }^{2}\right)$ Z.S.St., 26, p. 9, n. 3: »(On ne peut) a fortiori s'appuyer sur cette hypothèse gratuite pour déterminer la date de la loi Aebutia. Cela n'altère en rien d'ailleurs la valeur des autres raisons réunies par $\mathrm{M}$. Girard dans son beau travail sur la date de la loi Aebutia.« - ${ }^{3}$ ) Proceßgesetze, 2, pp. 187-188, où il dit que sacramento agere prouve seulement une procédure avec un enjeu qui est concevable sans être accompagnée de paroles sacramentelles s'accommodant à un texte de loi et où il présente comme pouvant s'entendre dans ce sens le langage de Puchta cité et critiqué par nous, Z.S.St., 14, p. 47, n. $1=$ N.R.H., p. 287, n. 1 . 
lés agissaient en répétition par la legis actio sacramenti. M. Cuq et M. Mitteis le reconnaissent tous deux. ${ }^{1}$ ) Or, cela reconnu, cette legis actio sacramenti ne serait compatible avec l'existence de la procédure formulaire que si l'on pouvait démontrer qu'il y avait ici une raison pour exclure la formule. On ne l'a pas fait. Le seul auteur qui l'ait tenté est $\mathbf{M}$. Mitteis qui a cherché la cause d'exclusion dans un rapprochement ingénieux avec les centumvirs et les décemvirs litibus iudicandis encore saisis par legis actio sous la procédure formulaire. Les jurys permanents ne sont, dit-il, jamais saisis par formules. C'est un rapprochement ingénieux. Mais d'abord e'est un rapprochement, ce n'est pas une preuve. Ensuite, c'est un rapprochement gratuit, car, d'un côté, pour pouvoir argumenter des actions réelles déférées aux centumvirs et aux décemvirs à notre action personnelle, il faudrait savoir pourquoi la procédure du sacramentum a été gardée pour ces actions réelles, et, d'autre part et surtout, il n'est pas établi que la legis actio sacramenti in personam des lois Calpurnia et Junia ait été jugée par un jury permanent du type des décemvirs et des centumvirs; il ne l'est pas même qu'elle l'ait été par le grand jury de la procédure développée des quaestiones perpetuae auquel pense évidemment M. Mitteis: l'existence de ce jury est prouvée pour les poursuites criminelles de la loi Acilia; elle ne l'est pas pour les réclamations civiles de la loi Calpurnia et de la loi Junia. ${ }^{2}$ ). Et les lois Calpurnia et Junia restent en conséquence, à notre avis, au début du $\mathrm{VII}^{\mathrm{e}}$ siècle de Rome, une sorte de borne chrono-

1) Cuq, Institutions, $1^{2}$, p. 285, n. 1. Mitteis, Privatrecht, pp. 124, n. 45. 132, n. $30 . \quad$ - 2) C'est un point que j'ai déjà souligné, Z.S.St., 14 , p. 45 , n. $1=$ N.R.H., 1897 , p. 285 , n. 1 . Pour que la quaestio créée par la loi Calpurnia ait eu le caractère de quaestio perpetua que lui attribue le texte de Cicéron, Brut., 27, 106, il suffit que le préteur pérégrin ait été chargé par la loi Calpurnia à titre permanent d'accueillir les réclamations des provinciaux victimes d'extorsion. Cela n'implique aucunement que le magistrat ait dès alors participé à la sentence, encore moins que le jury ait été un grand jury, comme celui de 50 membres tiré, à partir de la loi Acilia, de la liste de 450 jurés dressée d'après cette loi par le président de la quaestio, la première année encore par le préteur pérégrin et les suivantes par le nouveau préteur repetundis. 
logique au delà de laquelle aucune conjecture ne peut reculer la loi Aebutia.

II. On n'a chronologiquement que bien peu de chemin à faire dans l'autre sens pour descendre aux premières années où surgissent les vestiges de la loi nouvelle. Mais, avec ces vestiges, nous retrouvons les controverses qui d'ailleurs, pouvons-nous remarquer, ont beaucoup moins porté sur la matérialité des faits relevés par nous que sur les principes généraux en partant desquels nous en avons tiré des conséquences.

1. Le point où j'ai rencontré et où je savais parfaitement devoir rencontrer les contradictions les plus nombreuses et les plus animées est celui qui touche au droit du magistrat de refuser la legis actio. C'est, en lui-même, le moins essentiel pour mes chiffres positifs, qui en le négligeant, seraient demeurés identiques à deux ou trois ans près. Mais ce n'était pas une raison pour mutiler mon exposition en éliminant un élément de solution qui devait régulièrement y figurer; aujourd'hui encore j'aurai d'autant moins de scrupule à revenir sur lui avec quelque détail qu'il s'agit là, je crois, d'un principe fondamental pour la saine intelligence de l'ancienne procédure romaine.

J'ai écrit que, le magistrat du temps des Actions de la loi n'ayant pas le droit d'empêcher les parties d'intenter la legis actio, l'existence de la loi Aebutia me parâ̂t établie par le texte de Cicéron selon lequel deux avocats se disputaient, en 627 ou 628 , pour savoir si le magistrat prescrirait au demandeur d'intenter l'action qu'il voulait ou celle que voulait le défendeur ${ }^{1}$ ), et je penserai la même chose soit des deux textes de Cicéron et du Digeste selon lesquels la vindicatio in libertatem était refusée par l'édit de Drusus à l'affranchi qui ne renouvelait pas le iusiurandum de operis et par un édit déjà connu de Q. Mucius Scaevola à l'homme libre qui s'était laissé vendre pour partager le prix, soit de celui de Gaius selon lequel un édit antérieur à la loi Junia refusait la vindicatio in servitutem aux auteurs d'affranchissements

1) Cicéron, De orat., 1, 36, 166 (p. 116, n. 1). 
faits en dehors des formes ${ }^{1}$ ), comme d'ailleurs de tous les autres textes, qui attesteraient une pareille influence du magistrat sur l'exercice de l'action.

M. Huvelin a proposé, du texte de Cicéron sur le procès de $627-628$, une interprétation que je ne crois pas exacte ${ }^{2}$ ),

1) Cicéron, Ad Att., 7, 2, 8 (p. 116, n. 6). D., 40, 12, 23, pr. Gaius, 3, 56: Admonendi sumus ... eos qui nunc Latini Iuniani dicuntur, olim ex iure Quiritium servos fuisse sed auxilio praetoris in libertatis forma servari solitos. Cf. le fr. dit de Dosithée, 5. - 2) Dans la contestation entre Hypsaeus, avocat du pupille demandeur réclamant l'action où son client échouerait pour plus petitio, et Octavius, avocat du tuteur défendeur qui s'oppose à ce qu'on commette contre son client la plus petitio qui le libérerait ( $\mathrm{p} .116, \mathrm{n} .1$ ), on admet généralement qu'il s'agit non de l'action tutelae de la procédure formulaire, mais de l'action rationibus distrahendis au double de l'ancien droit donnée contre le tuteur infidèle à raison de son dol et non de ses fautes, sauf un peu plus d'hésitation sur le point de savoir si elle était intentée par sacramentum ou par iudicis postulatio dans la procédure des Actions de la loi à laquelle se rapporte le texte, sauf plus d'hésitation encore sur la nature de la plus petitio dont il s'agit. M. Huvelin, dont l'idée est nouvelle, a pensé, Mélanges Gérardin, pp. 319-354, que la discussion porterait non pas sur le point de savoir comment l'action rationibus distrahendis serait intentée, mais, après que cette action aurait été intentée par sacramentum et que le sacramentum du demandeur aurait été déclaré iustum, comment on intenterait l'arbitrium liti aestimandae; ce qui, dit-il, rend plus compréhensible que le demandeur s'expose à une plus petitio en voulant comprendre dans le second procès des chefs de créances étrangers au premier. Puis il part de là pour admettre une scission du même genre en matière d'autres actions telles que l'action furti nec manifesti, l'action iniuriarum, l'action aquae pluviae arcendae, où l'on agirait de même successivement par sacramentum et par arbitrium liti aestimandae et il cherche même, dans un écrit rédigé à l'occasion du passage du premier procès au second, l'origine de la procédure formulaire. A nous en tenir à notre texte, l'interprétation proposée par M. Huvelin ne nous semble pas admissible. La coupure imaginée par lui n'explique pas le texte mieux qu'on ne peut faire en dehors d'elle; car, que le procès initial s'intente par sacramentum ou par iudicis postulatio, l'idée selon laquelle le demandeur y réclame des articles qui ne rentrent pas dans le cadre de l'action, par ex. selon la conjecture ingénieuse de M. Lenel, Edictum ${ }^{2}$, 1907, p. 310, n. 4, à raison du mot captum, $D ., 27,3,1,23$, toutes les valeurs que le tuteur a prises, aussi bien celles qu'il a seulement dissipées que celles qư'il a soustraites, est aussi vraisemblable que celle où il demanderait dans le second procès des valeurs sur lesquelles n'aurait pas porté le premier. Mais, de plus la supposition qu'il s'agisse dans le texte d'un 
mais que je n'ai pas besoin de discuter ici; car elle n'empêcherait pas, en réalité, le texte d'impliquer une contestation devant le magistrat sur la délivrance de l'action et par contrecoup un pouvoir du magistrat sur l'action qu'il n'a acquis, à mon avis, que depuis la loi Aebutia. ${ }^{1}$ ) Mais c'est précisément ce défaut du droit du magistrat sur l'action dans la période antérieure à la loi Aebutia qui a été contesté soit à propos de ma doctrine, soit en dehors d'elle, non seulement par M. Wlassak, mais par plusieurs autres auteurs et sur lequel je dois m'expliquer ici.

Ce qu'on n'a pas le droit d'accorder, on n'a pas le droit de le refuser. Par conséquent, dans un système procédural où le magistrat n'a pas le droit d'accorder l'action, il n'a pas le droit de la refuser. Il n'a pas le droit d'accorder l'action, ou plus précisément d'autoriser à l'exercer valablement, quand la loi ne la donne pas, parce qu'elle ne vient pas de lui, mais de la loi, et c'est aussi parce qu'elle ne vient pas de lui, mais de la loi, qu'il n'a pas le droit de la refuser, ou plus exactement d'empêcher de l'exercer, quand la loi la donne. C'est là le droit de Rome qui demeurerait le droit, quand bien même on y apercevrait quelques infractions pratiques; mais cela a aussi été le fait du moins dans la période de la République qui va jusqu'à la loi Aebutia; car, si le fait et le droit ont pu impunément être mis en désaccord sous la royauté où le roi était rendu pratiquement irresponsable par le caractère viager de ses pouvoirs, les magistrats annaux de la République qui auraient contrevenu à leur devoir en empêchant l'exercice de legis actiones, en

second procès en estimation nous parait impossible pour plusieurs raisons. Pour n'en citer qu'une, si les deux avocats plaidaient sur un procès en liquidation de créances déjà reconnues, Cicéron ne pourrait pas dire que la plus petitio ferait éviter l'infamie au défendeur; car cette infamie résulterait nécessajrement pour le tuteur de ce qu'il aurait été reconnu coupable de détournement dans le premier procès, quoiqu'un incident postérieur le dispensât de payer sa dette.

1) M. Huvelin, p. 325, semble d'un avis différent; mais le débat même entre les avocats implique que le magistrat pourra forcer le demandeur à s'exprimer dans certains termes plutôt que dans d'autres, l'empêcher d'intenter son action comme il veut; or c'est précisément ce qu'il ne pouvait pas, à notre avis, avant la loi Aebutia.

Zeitschrift für Rechtsgeschichte. XXIX. Rom. Abt. 
les arrêtant par le refus de leur concours, auraient été atteints, à leur sortie de charge, à raison de ce manquement à leurs obligations professionnelles.

Les legis actiones sont des procédures d'un type très ancien qui se retrouve dans beaucoup d'autres législations archaïques. ${ }^{1}$ ) Ce sont des procédures dérivées de la justice privée, dans lesquelles celui qui prétend avoir un droit le fait valoir au moyen de gestes et de paroles sacramentels qui produisent un effet propre et nécessaire s'ils ne sont pas arrêtés par d'autres gestes et d'autres paroles également sacramentels. Depuis une époque ignorée, ces gestes sont faits, ces paroles sont prononcées devant le magistrat auquel le rituel indique les gestes qu'il doit lui-même faire, les paroles qu'il doit lui-même prononcer, selon que les parties en ont fait ou prononcé d'autres, ainsi selon que la demande a été ou non régulièrement contestée. Il devra par exemple, selon que les parties auront ou non fait ce qui est nécessaire pour lier un procès, organiser ou non l'instance qu'il tranchait lui-même à l'époque la plus ancienne, dont il doit renvoyer la solution à un juré depuis une époque plus récente, mais encore fort reculée. Mais l'activité du magistrat est commandée par celle des parties. C'est l'activité des parties qui l'amène à renvoyer l'affaire au juré ou plus anciennement à la trancher, quand elles ont fait ce qu'il faut pour cela. Auparavant c'est l'activité des parties qui l'amène à prononcer certaines paroles, quand elles en ont prononcé d'autres. Il n'a pas à se demander s'il doit ou non renvoyer l'affaire au juré, quand le cérémonial nécessaire a été accompli. C'est un devoir qui nait pour lui de l'accomplissement de ce cérémonial. Il n'a pas non plus auparavant à se demander s'il doit prononcer les paroles appelées par l'activité des plaideurs à mesure que cette activité se réalise, par exemple les mots mittite ambo hominem quand les deux plaideurs ont procédé à la vindicatio et à la contravindicatio mobilières ${ }^{2}$ ), par exemple les mots ite viam, inite viam, dans la revendi-

1) $\nabla$. par ex. pour l'ancien droit germanique, l'exposition et les renvois de Schröder, Lehrbuch der deutschen Rechtsgeschichte ${ }^{7}$, 1907, pp. 84-90; cf. Brunner, Deutsche Rechtsgeschichte, 1², 1906, pp. 251-267.

$\left.-{ }^{2}\right)$ Gaius, 4, 16. 
cation des immeubles, à propos de laquelle un texte de Cicéron, probant entre tous, souligne son asservissement au rituel. ${ }^{1}$ ) Il n'a pas davantage à se demander s'il doit ou non les laisser entamer le cérémonial de la legis actio, quand ils arrivent devant lui. La loi lui a donné une tâche et non pas une autre. Et il sortirait de sa tâche, il ferait ce qu'il n'a pas le droit de faire en même temps qu'il commettrait un déni de justice, en refusant de renvoyer une affaire correctement liée devant un juré, en ne répondant pas aux paroles de l'une ou l'autre partie par la formule que prescrit le rituel, ou encore en empêchant par je ne sais quel jeu de cache-cache l'un ou l'autre plaideur, en particulier le demandeur, de prononcer efficacement devant lui les paroles que ce plaideur veut prononcer. Le magistrat n'a pas à s'occuper du bien fondé de la demande avant que le procès soit lié par la legis actio, pas plus à l'époque où il statue lui-même qu'à celle où c'est le juré qui statue. En le faisant sous prétexte d'apprécier la légalité ou la moralité de l'instance, il commettrait un empiètement sur les pouvoirs du juré à la deuxième époque, il fausserait, à la première comme à la seconde, les rouages de la procédure par un coup de force.

Et ces idées résultent si naturellement de l'ensemble des choses qu'elles ont toujours été implicitement ou explicitement admises. Par un mode d'argumentation aussi flatteur pour moi que désavantageux pour elles, M. Wlassak paraît, en dirigeant sa discussion presque exclusivement contre moi, les présenter comme m'ayant eu pour ainsi dire comme seul partisan. En réalité, tout ce que j'ai fait, cela a été d'en tirer pour un problème spécial une conclusion à laquelle on n'avait pas songé et peut-être en conséquence d'insister sur elles avec une énergie particulière. Il suffit de se reporter aux notes de M. Wlassak pour voir qu'elles ont été au contraire émises avant moi avec une égale netteté par quelquesuns des premiers romanistes du $\mathrm{XIX}^{\mathrm{e}}$ siècle, ainsi, pour ne

1) Cicéron, Pro Murena, 12, 26: Praetor interea ne pulchrum se ac beatum putaret atque aliquid ipse sua sponte loqueretur, ei quoque carmen compositum est, cum ceteris rebus absurdum tum vero in illo. 
citer que trois grands noms, par de Jhering ${ }^{1}$ ), par Mommsen ${ }^{2}$ ) et par Pernice. ${ }^{3}$ ) Mais il y a mieux. Ce n'est pas seulement par ces maîtres que le caractère du rôle joué par le magistrat sous les Actions de la loi a été aperçu. C'est, pourrait-on dire, par tous ceux qui ont écrit sur la procédure

1) Il suffira de reproduire parmi les nombreux passages du $\$ 43 \mathrm{c}$ qu'a cités M. Wlassak et dont ou pourrait encore augmenter la liste, celui qui exprime le plus éloquemment les idées mêmes dont nous nous sommes inspiré (Geist des römischen Rechts, 2, $2^{\text {s }}, 1889=\mathrm{tr}$. fr. 3, 330): Es steht unter allen Kundigen fest, daß mit dem Formularproze $\beta$ eine neue Ära für das römische Recht beginnt, nicht etwa bloß für den Prozeß, sondern auch für das materielle Recht. Erst mit diesem Zeitpunkt fängt jene Rechtsquelle an zu fließen, die für das ganze Recht eine Quelle neuen Lebens werden sollte, das prätorische Edict ${ }^{896}$. Es ist nicht Zufall, daß dies erst jetzt geschah; das Legisactionensystem und das Edict waren unverträglich miteinander. Im alten Prozeß waren dem Prätor die Hände gebunden, er war nichts als ein Stück der Maschine ${ }^{807}$, er in ihrer Gewalt, sie nicht in der seinigen; erst das Formularverfahren gewährte jene Freiheit der Bewegung, welche die wesentliche Bedingung seiner rechtsbildmerischen Thätigkeit bildete. Et il cite, aux notes 896 et 897, les deux textes de Gaius, 4, 11, et de Cicéron, Pro Mur., 12, précisément dans le sens où je les cite, p. 150, n. 1, et p. 131, n. 1. - ${ }^{2}$ ) Th. Mommsen, Römisches Staatsrecht, 3, 1887, $59=$ tr. fr., $6,1,1889,64$, et de nombreux autres passages relatifs en particulier à la juridiction grâcieuse. - ${ }^{3}$ ) A. Pernice, Z.S.St., 14, 1893, p. 143, n. 5 , cité par Wlassak, mais aussi $Z . S . S t$., 20, 1899, p. 131, n. 5, par ex. Il ne serait d'ailleurs pas très difficile d'allonger la liste donnée par M. Wlassak, 25, p. 81, n. 2, des partisans de la doctrine qu'il réprouve. Ainsi il aurait pu citer, à côté de M. Jörs, Römische Rechtswissenschaft, 1, 1888, pp. 157. 196 et ss., Baron, Institutionen u. Civilprozeß, 1884, p. 382, n. 3, qui repousse formellement le droit de denegatio legis actionis en citant Val. Max. 7, 7 (p. 140, n. 1) comme une exception qui est un déni de justice. De même il faudrait y classer ceux qui ont adopté ma doctrine sur la date de la loi Aebutia, sans faire sur ce point particulier de réserve implicite ou explicite, non seulement M. Giffard, La confessio in iure, 1900, pp. 17-37, n. 2, que M. Wlassak nomme, tome 25, p. 81, n. 2, et M. Boulard, Les instructions écrites $d u$ magistrat au juge commissaire, 1906, p. 2, n.1, qu'il censure, tome 28, p. 98, n. 2, mais M. Senn, Leges perfectae minus quam perfectae et imperfectae, 1902, pp. 3 et ss., 73, n. 3; M. Fliniot, Le vadimonium, 1908, p. 36, n. 1 ; M. N. Herzen, Précis de droit romain, Lausanne, 1906, pp. 115. 116; M. Gaston May, Eléments de droit romain", 1907, p. 581, et sans doute M. Seckel, qui dans son édition de Heumanns Handlexikon zu den Quellen des römischen Rechts ${ }^{2}, 1907$, p. 21, v. Aebutia lex, place la loi avec nous entre l'an 605 et l'an 628 . 
romaine, aussi bien par exemple par Bethmann-Hollweg que par Keller de qui M. Wlassak remarque lui-même qu'il a écrit que l'ancienne procédure contient des paroles sacramentelles der Parteien, wodurch die Aussprïche des Magistrats in ebenso bestimnter Wortfassung gleichsam hervorgerufen wurden ${ }^{1}$ ), pour ne pas parler de presque tous les modernes de qui M. Wlassak a soigneusement relevé l'adhésion au droit de denegare legis actionem. Ces auteurs en général ${ }^{2}$ ) ne méconnaissent pas plus que Mommsen, Pernice ou de Jhering la façon dont le rituel des legis actiones s'impose au magistrat en même temps qu'aux parties. Toute la différence est que, sans nier le principe, ils lui admettent des atténuations en vertu de ces vraisemblances apparentes qui sont toujours si dangereuses pour l'intelligence d'institutions un peu originales et fortes. Sous l'influence à la fois $\mathrm{du}$ régime de l'époque formulaire et de certaines habitudes d'esprit modernes, on a pensé que la règle devait fléchir plus ou moins largement dans certains cas. Mais c'est l'erreur contre laquelle je me suis élevé, et que je dois combattre encore. Quand une conception juridique, religieuse, économique, politique quelconque apparait clairement

1) Keller, § 12. La trad. française de Capmas, 1870, p. 46, ne reproduit pas là le sens du texte. Cf. Bethmann-Hollweg, Civilprozeß, 1, 1864, p. 52. - 2) V. par ex. Karlowa, Civilprozeß zur Zeit der Legisactionen, 1872, p. 3: "Auch der Magistrat ist, soweit er beim lege agere mitwirkt, streng durch den Buchstaben der Lex gebunden, wenn ihm durch die Lex selbst nicht ein Ermessen verstattet ist... Er hat zu sprechen, was im Anschluß an die Lex die pontificische Jurisprudenz an Formeln für ihn ausgebildet hat... Der Magistrat war als Theilnehmer am lege agere nichts weiter als ein Stück der Prozeßmaschinerie, ihm waren die Hände vollständig gebunden «, et encore p. 342 (où il admet la possibi'lité d'un refus de concours en s'appuyant sur Cicéron, De orat., 1, 36, 166, p. 116, n. 1): Dieselbe Befugnis (de dare ou de denegare legis actionem) dem Prätor auch für die Legisactionen einräumen zu wollen, hieße, wie man mit Recht gesagt hat, die Existenz des Legisactionenprozesses in Frage stellen. C'est aussi à peu près forcément le point de vue de ceux qui ont adopté mon raisonnement d'ensemble sur la date de la loi Aebutia tout en admettant la possibilité d'un refus de concours du magistrat, implicitement comme M. Partsch (p. 147, n. 1) ou explicitement comme M. Erman, Z.S.St., 19, 1889, pp. 275. 285, n. 2 rapproché de Z.S.St., 14, p. 246, n. 2 cité par Wlassak. 
dans un milieu, particulièrement dans un milieu peu avancé en évolution, ce qu'il faut. dire, ce n'est pas qu'elle y a figuré avec les atténuations, les restrictions, les tempéraments qui la rendent supportable pour nos idées à nous. C'est qu'elle a dû au contraire, à un moment donné, sortir tous ses effets, produire toutes les conséquences, bonnes ou mauvaises, naturelles ou surprenantes, qu'elle portait en elle. Les atténuations, les restrictions, les tempéraments, sont choses du déclin de l'institution, du temps de sa décadence, du moment où les conceptions dont elle procédait s'affaiblissent. La seule question serait de savoir quand cet affaiblissement est arrivé pour cette notion de la legis actio qui excluait le droit du magistrat de lui dénier son concours. Or, nous avons, à mon avis, la preuve qu'il ne s'est pas produit avant l'introduction de la procédure formulaire, qu'en particulier il ne s'était pas encore produit au $\mathrm{VI}^{\mathrm{e}}$ siècle de la ville, et nous pouvons même, je crois, dire pourquoi.

La preuve que le système existait encore intact à la fin du $\mathrm{VI}^{\ominus}$ siècle, à la veille de l'introduction de la procédure formulaire, est dans les multiples leges imperfectae ou minus quam perfectae ${ }^{1}$ ), qu'on voit alors poursuivre par le mécanisme incommode d'une action en répétition le résultat qui a plus tard été atteint plus simplement au moyen d'une exception et qui eût été dès alors obtenu sans peine à l'aide d'un refus d'action si le magistrat avait eu le droit de denegare legis actionem. Quand, dans la seconde moitié du VIe siècle, on a voulu protéger le mineur de vingt cinq ans contre les engagements arrachés frauduleusement à son inexpérience, l'héritier testamentaire contre des legs trop élevés, on aurait pu, semble-t-il, charger le magistrat d'arrêter les actions intentées à l'encontre de la loi Plaetoria, de la loi Furia de legatis. ${ }^{2}$ ) Au lieu de cela, la loi Plae-

1) V. Senn, Leges perfectae, minus quam perfectae et imperfectae, 1902 et la trad. allemande de notre Manuel, Geschichte und System des römischen Rechts, 1908, p. 1023, n. 1. L'article de M. Baviera, Studi giuridici in onore di C. Fadda, Napoli, 1906, 2, pp. 205-219, est presque exclusivement relatif à l'origine de la distinction. - 2) La loi Plaetoria qui donne au mineur contre celui qui l'a trompé une action en répétition visée loi Julia de 709, ligne 111, D., 4, 4, 24, 3 et fr. de 
toria, la loi Furia de legatis, d'autres encore que je ne cite pas pour ne pas compliquer le débat de polémiques indépendantes, ont recouru à l'expédient gauche et compliqué d'actions données contre celui qui a agi d'après la loi ancienne à celui qui n'aurait pas dû être poursuivi d'après la loi nouvelle. Ce serait inexplicable si le magistrat n'était pas même alors dépourvu de tout pouvoir d'arrêter l'action. Sans doute, M. Wlassak, généralisant hardiment une idée émise pour la loi Furia de sponsu par M. Appleton, soutient que l'on aurait eu partout le choix entre le droit de répéter et celui de refuser de payer, et il invoque des textes de l'époque classique qui montreraient que, pour certaines espèces d'intérêts usuraires par exemple, on a le choix entre le refus de paiement et la répétition. ${ }^{1}$ ) Mais comment argumenter du droit opulent et touffu du Principat au droit sobre et pauvre de la République? Qui ne voit que l'idée de cumul, proposée par M. Appleton ${ }^{2}$ ) pour la seule loi Furia où elle parait déjà bien invraisemblable ${ }^{3}$ ), devient d'une impossibilité criante quand on l'étend partout? Que devien-

formula Fabiana, 4 (Manuel, p. 228 , n. $3=$ tr. all. p. 252, n. 6), est citée comme récente par Plaute dans des pièces représentées en 563 ou vers 563, Pseudulus, 1, 3,69-70. Rudens, 5, 3, 24-26 (Manuel, p. 227, n. 2, trad. p. 252, n. 1). La loi Furia testamentaria qui donne à l'héritier contre le légataire qui a reçu plus de mille as un manus iniectio pura en répétition (Gaius, 2, 225. 4, 23) est postérieure à la loi Cincia de 550 (p. 166, n.3) d'après son classement des personnes exceptées, à montré Bruns, Z.R.G., 12, 1876, p.133, et d'après le langage de Gaius, 2, 225-226, elle est antérieure à la loi Voconia de 585 (p. 166, n. 5).

1) D., 12, 6, 26, 1. Cf. D., 12, 6, 26, $p$. M. Wlassak invoque encore le droit de choisir entre l'action contraria et la compensation opposée ex eadem causa sur l'action directa dans les contrats ou quasi contrats synallagmatiques imparfaits (où il ne date que de la création des formules in ius) et celui d'opter entre l'action et l'exception metus (dont il n'a pu être question qu'à partir du jour probablement postérieur à la création de l'action, où l'exception a existé, et même depuis celui plus récent encore où l'action a cessé d'être considérée comme purement pénale. Cf. Gradenwitz, Ungültigkeit obligatorischer Rechtsgeschäfte, 1887, p. 37 et ss.; Manuel, p. 416, n. 1 et trad. p. 454, n. 3). - 2) Z.S.St., 26, 1905, pp. 1-48. Mélanges Gérardin, 1907, pp. 1-19. ${ }^{-3}$ ) V. nos observations sur le premier article Manuel, p. 756, n. 1; Studi in onore di Fadda, Napoli, 1906, 2, pp. 59-62, et sur le second, Geschichte und System, 1908, p. 825, n. 3. 
drait, avec cette surabondance de moyens, ce trait caractéristique de l'ancien droit que de Jhering appelait la loi de l'économie juridique?

Les actions en répétition des leges imperfectae et minus quam perfectae sont le plus éclatant témoignage du maintien intégral, jusqu'aux temps les plus récents du système, de la règle primitive qui défendait au magistrat d'arrêter l'exercice d'une Action de la loi, et ce maintien intégral s'explique à son tour très bien par une règle constitutionelle dont le refus de la legis actio aurait été la violation. Cette règle constitutionnelle, c'est la règle, rattachée par la tradition romaine aux fondateurs de la République, en tout cas antérieure aux XII tables, selon laquelle le magistrat est non pas autorisé, mais obligé à renvoyer le jugement des procès civils aux jurés. ${ }^{1}$ ) Ainsi que nous l'avons déjà remarqué, le magistrat, en examinant d'une manière quelconque le bien fondé de la legis actio avant de laisser le demandeur entamer en face du défendeur le cérémonial qui liera le procès ou le rendra inutile, aurait, de tous les temps, apporté une perturbation grave à la marche de la procédure dans laquelle la legis actio doit venir avant l'examen de l'affaire; mais, depuis la division de l'instance entre deux autorités, il commettrait par là une usurpation sur les pouvoirs du juré, une violation flagrante de la règle constitutionnelle qui a établi le système du jury civil comme une garantie contre l'arbitraire du magistrat.

Cette barrière n'a cédé que sous une secousse qui n'a pas été sans ébranler la règle constitutionnelle elle-même, que par le contrecoup de la loi Aebutia.

Comment la loi Aebutia a-t-elle entamé cette substitution des formules aux Actions de la loi qui fut terminée seulement par les lois Juliae? Suivant l'interprétation, à mon avis très juste, de M. Wlassak, elle a mis à l'essai la procédure à laquelle les lois Juliae ont, l'expérience une fois faite, donnée la prédominance exclusive. Elle a permis aux parties sous le contrôle du magistrat de choisir entre la

1) V. Organisation judiciaire, 1, pp. 77-82. Cf. M. Wlassak luimême, Prozeßgesetze, 2, pp. 328-346. 
procédure ancienne et la procédure nourelle. Par conséquent, en définitive, elle a permis au magistrat, dans chaque cas concret, ou bien de laisser le procès s'organiser selon l'ancien système, par les gestes et les paroles des parties, alors naturellement sans délivrer de formule, ou bien au contraire de délivrer une formule, alors naturellement en ne laissant pas intenter la legis actio, en résumé d'accorder à son gré la legis actio ou la formule, en refusant la legis actio quand il accordait la formule et la formule quand il accordait la legis actio. C'est ce que $\mathbf{M}$. Wlassak a très finement aperçu et démontré. Mais M. Wlassak n'a pas vu et se refuse à reconnaitre une conséquence de son système qui donne à ce système une importance très supérieure à celle de la découverte d'une curiosité d'histoire procédurale. ${ }^{1}$ ) C'est que le mécanisme qu'il a mis en lumière explique merveilleusement l'influence décisive que la doctrine commune assignait depuis longtemps à la loi Aebutia, non pas simplement sur la procédure, mais sur tout le développement postérieur du droit, sans pouvoir à la vérité parfaitement en discerner les causes.

La loi Aebutia a permis au préteur d'accomplir tout cet ensemble de créations véritablement législatives qu'on rassemble sous le nom de droit prétorien, précisément parce que, sans doute d'une façon plus accidentelle que réfléchie, elle a donné pour la première fois au préteur le pouvoir d'accorder ou de refuser les actions. Pour créer tout le droit prétorien, il a suffi au préteur d'utiliser isolément les

1) Je peux répéter ici une observation faite p. 121, n. 1. M. Wlassak a proposé en 1888, Prozeßgesetze, 1, pp. 66-72. 85-103, du problème du partage de la réforme entre la loi Aebutia et les lois Juliae, une solution qui m'a paru d'une rare élégance. Je l'ai franchement acceptée l'un des premiers, sinon le premier, N.R.H., 1889, p. 298. Seulement je lui associe des idées qui à mon avis en sont le principal intérêt et que $M$. Wlassak n'avait pas eues, qu'il repousse vivement. Je peux avoir tort ou raison. Mais le fait que la découverte première dont nous discutons les conséquences est due à M. Wlassak ne fait rien à la chose. Cela donne à M. Wlassak un mérite personnel qui ne pourra pas lui être enlevé. Mais cela ne fait absolument rien pour la vérité ou la fausseté de l'interprétation donnée par d'autres ou par lui des faits qu'il a mis en lumière. 
deux pouvoirs qu'il n'eut dû correctement employer que simultanément: d'accorder la formule quand il n'avait pas de legis actio à refuser et de refuser la legis actio quand il n'accordait pas de formule. C'est la loi Aebutia qui pour la première fois a donné au magistrat le pouvoir d'accorder des actions, en visant le cas où il accorderait une formule à la place de la legis actio déjà donnée par le droit, mais en brisant la barrière qui était entre l'action et lui, en lui ouvrant la possibilité matérielle d'accorder des actions que la loi ne donnait pas, par la délivrance en dehors des cas légaux, de formules fictices, de formules avec transposition et de formules in factum. C'est pareillement la loi Aebutia qui pour la première fois a pourvu le magistrat du droit de refuser l'action, qui lui a permis d'arrêter l'exercice de la legis actio, de denegare legis actionem, en visant encore le cas où il donnerait à la place une formule, mais en brisant encore la barrière qui était auparavant entre lui et l'action, en lui ourrant la possibilité matérielle de paralyser l'action légale quand il userait de son pouvoir de refuser la legis actio sans délivrer de formule. Assurément il y a là toujours une dérogation indirecte au principe de la compétence exclusive du juré en matière de jugement auquel on rencontre depuis la loi Aebutia d'autres infractions encore. Assurément aussi et plus largement une opinion publique et des pouvoirs de l'Etat suffisamment vigilants auraient pu arrêter le préteur à ses premiers pas dans la voie de cette application extensive et sûrement abusive de la loi. Mais c'est seulement la loi qui a fourni aux empiètements leur instrument.

A la vérité, les derniers auteurs qui ont soutenu que le magistrat n'a pas attendu la loi Aebutia pour obtenir le droit de denegare legis actionem ont invoqué deux ordres d'arguments: un principe théorique qui conduirait à lui reconnaitre ce droit dès le temps les plus reculés et des exemples concrets qui attesteraient qu'il le possédait tout au moins avant la loi Aebutia. Mais je crois que l'on se trompe sur les exemples et sur la règle.

La règle qui exigerait que le magistrat ait eu, sous les Actions de la loi et, semble-t-il, dès les temps les plus 
reculés, le droit d'arrêter la legis actio serait, d'après M. Wlassak, le principe posé par l'illustre Mommsen selon lequel tout le développement interne de l'Etat romain se ramènerait à l'enchainement croissant de la magistrature qui devrait donc avoir été encore plus libre de refuser l'action à l'époque ancienne qu'à l'époque moderne. ${ }^{1}$ ) M. Mommsen lui-même aurait dit cela en présentant les faits positifs concrets comme devant être effacés par une règle générale abstraite que, malgré mon respect et mon admiration pour le maître, je resterais avec la bonne méthode et la vérité contre lui, pour les faits concrets positifs contre la règle abstraite. Mais en réalité, M. Mommsen n'a pas dit cela. Il a même dit juste le contraire; car M. Wlassak, qui invoque si solennellement son autorité pour tirer d'un principe général posé par lui une conséquence qu'il n'en a jamais tirée, le tance assez vivement à quelques pages de distance pour avoir admis comme moi et même, note $M$. Wlassak, plus largement que moi, en matière de juridiction volontaire, l'idée que le magistrat n'a pas le droit de denegare legis actionem. ${ }^{2}$ ) Je peux même ajouter que cela n'empêche pas dans notre conception la magistrature d'avoir été, quant à la denegatio legis actionis, plus libre à l'origine, qui est le temps des rois, qu'à l'époque postérieure, qui est le temps de la République. Il est vrai que, dans notre idée, le magistrat judiciaire a eu, quoi qu'on pense de la courbe générale, une liberté plus grande après la loi Aebutia qu'arant. Mais M. Wlassak lui-même aurait de la peine à prouver que la loi Aebutia ait restreint les pouvoirs du magistrat judiciaire.

Restent les exemples concrets, dont la présence serait probante pour moi comme pour tout le monde, mais dont

1) Mommsen, Römisches Strafrecht, p. 341 (= tr. fr. 2, p. 4) relativement à la coercition des magistrats: "Die gesammte innere Entwickelung des römischen Gemeinwesens auf die Fesselung des Imperiums durch das Gesetz hinauslaufend « cité par Wlassak, 25, p.131, n. 2, où il renvoie aussi à la p. 171 (= tr. fr. 1, p. 197), relative à la justice criminelle des comices. Cf. Mitteis, p. 49, où il invoque seulement en général la notion de l'imperium du magistrat dégagée par Mommsen. - 2) Wlassak, 28, p. 91, n. 1. V. plus haut la p. 132, n. 2. 
l'absence est précisément une nouvelle preuve du système primitif.

On citait toujours autrefois un texte de Valère Maxime qui représente un magistrat refusant la legis actio dans une affaire de bonorum possessio. ${ }^{1}$ ) Comme j'ai remarqué il y a déjà longtemps, rien ne prouve que ces faits soient antérieurs à la loi Aebutia et il y a des raisons pour qu'ils lui soient postérieurs.

M. Mitteis s'est demandé récemment ${ }^{2}$ ) si l'exemple. cherché ne pourrait pas être trouvé dans le vetus edictum Drusi sur la denegatio de la vindicatio in libertatem à l'affranchi. ${ }^{3}$ ) Je réponds pareillement que rien ne prouve que les faits soient antérieurs à la loi Aebutia et qu'il y a même des raisons de les considérer comme un des témoignages de l'existence de la loi Aebutia; car, ainsi que j'ai dit et que l'admet M. Mitteis lui-même, le Drusus cité là est sans doute celui que la Rhétorique à Herennius cite en matière de mandat, lequel est sûrement le tribun de l'an 632 , consul en 642 , préteur au plus tard en 639 . Si c'est le même, il est une des preuves de la loi Aebutia. Sinon, il n'est pas daté et ne prouve rien.

Pour M. Wlassak, il a produit, en des lieux différents de son second article jusqu'à trois exemples dont je ne diminuerai pas la force probante en les rassemblant ici.

Le premier qu'il signale dans la longue note 1 de sa page 98 comme »m'ayant peut-être troublé d'un doute « serait dans la loi osque de Bantia que j'ai écrit avoir été probablement rédigée avant la loi Aebutia et sous l'influence romaine et qui prescrit au magistrat local, sous la menace d'une multa, de ne pas différer de plus de dix jours l'accom-

1) Val. Max., 7, 7, 5: Egregia Gaii quoque Calpurni Pisonis praetoris urbis constitutio. Cum enim ad eum Terentius ex octo filiis, quos in adulescentiam perduxerat, $a b$ uno in adoptionem dato exheredatum se querellam detulisset, bonorum adulescentis possessionem ei dedit heredesque lege agere passus non est. Cf. Z.S.St., 14, p. 31 , n. $3=$ N.R.H., 1897, p. 271, n. 3; v. plus haut p. 132, n. 3. - 2) Mitteis, Römisches Privatrecht, p. 140, n. 2. - ${ }^{3}$ ) Cicéron, $A d A t t ., 7,2,8$ (p. 116, n. 6). M. Wlassak a soumis ce texte à un examen approfondi dans la seconde partie de son travail, Z.S.St., 28, pp. 54-60. 
plissement d'une legis actio. ${ }^{1}$ ) M. Wlassak me demande comment je peux me soustraire à ce témoignage qui autorise le magistrat à refuser la legis actio, pendant les dix jours, absolument, et même après, en payant l'amende. Je réponds simplement qu'on ne doit pas confondre un texte destiné à maintenir dans des limites raisonnables les retards apportés par des obstacles de fait à l'accomplissement de la legis actio arec une disposition autorisant le magistrat à refuser systématiquement son concours à la legis actio et qu'il est d'une méthode encore plus défectueuse de considérer comme autorisant des actes les lois qui les interdisent sous peine d'amende: ce n'est tout de même pas, j'imagine, pour autoriser le vol non manifeste que la loi des XII tables l'a puni de la peine du double.

Aux pages 90 et 107, M. Wlassak invoque »un témoignage absolument inattaquable du droit du préteur de refuser la legis actio«. C'est le sénatus-consulte encore signalé par moi que Tite-Live rapporte avoir, en l'an 577, prescrit aux magistrats de ne concourir aux affranchissements qu'après que leur auteur aurait juré que l'affranchissement n'avait pas lieu civitatis mutandae causa. ${ }^{2}$ ) Je n'objecterai pas qu'il s'agit là de juridiction grâcieuse et non de juridiction contentieuse; car, à mon avis, les règles ont été, sous ce rapport, les mêmes pour toutes les legis actiones jusqu'à la loi Aebutia. Mais j'ai déjà remarqué, - en toute naïveté, comme dit M. Wlassak -, que, pour qu'il y eut là une preuve du droit du préteur, il faudrait que le préteur agisse spontanément et non pas sur l'ordre du Sénat. A cette époque où le Sénat était devenu pratiquement le premier pouvoir de l'Etat, les magistrats, habitués à recevoir ses injonctions, procédaient, quand ils étaient couverts par ses ordres, en matière judiciaire comme partout, à des actes qu'ils ne se seraient jamais permis de leur seule initiative.

Enfin M. Wlassak proclame aux pages 109 et 110, que j'ai involontairement reconnu un cas d'existence du droit

1) Loi osque de Bantia, ligne 23-27 (Bruns, Fontes ', pp. 52-53; Girard, Textes ${ }^{3}$, p. 29). Cf. Organisation judiciaire, 1, pp. 198-200. 2) Tite-Live, 41, 9, 10-11. Cf. Organisation judiciaire, 1, p. 195, n. 1. 
d'accorder et de refuser les actions avant la loi Aebutia. Ce serait en regardant comme prouvé, notamment par Gaius, un concours électif entre la legis actio sacramenti et la legis actio per condictionem ${ }^{1}$ ); car, dit M. Wlassak, »ce serait une lourde faute, d'une importance décisive pour la conception de l'ancienne procédure que de considérer comme indifférent, par exemple pour le demandeur qui choisit la legis actio sacramenti, que son adversaire accepte ou repousse son choix «; et en conséquence M. Wlassak, qui a la charité de ne pas vouloir comprendre dans le sens de cette doctrine fautive le passage de mon article où j'ai touché la question, m'offre sa propre doctrine selon laquelle, au cas de désaccord des parties, une décision du magistrat aurait été aussi nécessaire alors sur le point de savoir si on agirait par sacramentum ou par condictio que depuis la loi Aebutia sur celui de savoir si on agirait par legis actio ou par formules. Mais je dois décliner ce présent. Je n'ai, ni volontairement, ni involontairement, placé avant la loi Aebutia le droit d'accorder et de refuser l'action; car jai voulu dire et j'ai dit dans mon article, précisément ce que M. Wlassak considère comme une erreur si profonde et si préjudiciable. Je crois fermement que, dans le sacramentum in personam et dans le condictio comme dans l'ensemble des legis actiones, le demandeur qui a conduit le défendeur in ius et qui continue contre lui sa procédure devant le magistrat sans que l'autre y résiste d'une manière correcte, obtient sans jugement la satisfaction qu'en cas de résistance régulière il aurait obtenue en vertu du jugement, c'est-à-dire, sans autre formalité, s'il s'agit d'une créance d'argent, et après une procédure de liquidation accessoire, s'il s'agit d'une créance d'autre chose, le droit de procéder au bout du délai légal à la manus iniectio. Suivant le principe qui a été si bien dégagé par Demelius ${ }^{2}$ ), le même résultat est alors produit

1) Gaius 4, 95, qui montre que la sponsio faisait naitre une action sacramenti à côté de la condictio, comme d'ailleurs la loi Calpurnia de repetundis, p. 115, n. 4, fournit la même preuve pour la res; cf. Z.S.St., 14, p. 19, n. 1 = N.R.H., 1897, p. 257, n. 1, et aussi N.R.H., 1895, p. 421 et ss. Organisation judiciaire, 1, p. 188, n. 1. - $\left.{ }^{2}\right) \mathrm{V}$. Demelius, Confessio im römischen Civilprozeß, 1880, pp.39-110. A. Giffard, 
par le défaut de contestation régulière de la demande, par la confessio in iure expresse et par le jugement. C'est toujours vrai, d'après la loi Rubria, sous la procédure formulaire, pour les actions pour lesquelles la confessio équivaut encore au jugement et par corrélation le défaut de défense régulière à la confessio. ${ }^{1}$ ) C'est vrai pour toutes, sous la procédure des Actions de la $\operatorname{loi}^{2}$ ), par une conséquence naturelle

La confessio in iure, 1900 , pp. 7-49. M. Wlassak qui n'est pas beaucoup plus tendre pour M. Demelius, 25 , pp. 118, n. 4, 145-152, que pour les autres auteurs dont les solutions se trouvent mettre ses constructions en péril, est cependant obligé de reconnaître que la conception générale du livre n'a guère été contestée et qu'elle a rencontré des adhésions assez nombreuses (en particulier, dit-il, dans la science française, mais, suffiraient à montrer ses citations, non moins fréquemment dans la science allemande). En dehors de certaines particularités terminologiques (v. par ex. Wlassak, p. 118, n. 4) et de points de détail dont il ne faudrait pas exagérer la portée, son idée maitresse nous semble toujours d'une lumineuse évidence qui en explique la rapide propagation. Nous n'arons pas pour notre sujet à discuter l'étude approfondie à laquelle M. Wlassak a soumis la théorie de l'in jure cessio; mais il ne nous parait pas plus qu'à M. Mitteis, Privatrecht, p. 276, n. 42, être parvenu à démontrer qu'elle ne soit pas un procès simulé.

1) M. Wlassak déclare, 25, p. 150, n. 1, que le c. 21 de la loi Rubria contient rraisemblablement un droit récent et honoraire, puisqu'il assimile les faits de confiteri et de se non defendere et que les XII tables n'assimilaient à la chose jugée que la confessio. Mais l'idée que c'est la même chose de ne pas se défendre régulièrement ou d'avouer n'est pas seulement plus conforme à la physionomie de l'ancien droit qu'à celle du nouveau; la preuve qu'elle y remonte résulte des textes cités à la note suivante. - ${ }^{2}$ ) Cette doctrine s'appuie en première ligne sur le puissant témoignage d'ensemble que Demelius a tiré de la physionomie générale de l'ancienne procédure. Mais il ne faudrait pas croire qu'elle soit aussi dénuée d'appui dans les textes que le dit M. Wlassak, p.151, n. 3. Je citerai comme exemple les fragments de Valerius Probus (dans les formules de legis actiones), 4, 3: Quando neque ais neque negas et de Paul, $D$., 50, 17, 142: Qui tacet non utique fatetur: sed tamen verum est eum non negare. M. Wlassak les écarte tous deux en disant: pour le second, que la défectuosité de l'argument saute aux yeux, pour le premier, que son sens est indécis et qu'en particulier on ne peut le rapporter à la legis actio in personam parce que les $n^{\circ} 1$ et 2 de Valerius Probus y sont relatifs, car les nos 4 et 6 se rapportent à la vindicatio et le no 5 à une procédure pénale (ce qui d'ailleurs n'est pas prouvé; v. en sens contraire Mommsen, Strafrecht, p. 183, n. 3), 
de l'origine et du caractère même de la plus ancienne procédure. Et je pourrai dire à mon tour que je considère l'admission de l'idée contraire »comme une lourde faute, d'une importance décisive pour la conception de l'ancienne procédure«. En tout cas, cela fait que je considère le dernier exemple de denegatio legis actionis imaginé par M. Wlassak comme encore plus chimérique que tous ceux qui ont été proposés auparavant par lui ou par d'autres.

$\mathrm{Au}$ fond, d'ailleurs, si M. Wlassak multiplie tant les objections, les argumentations et les raisonnements de toutes catégories contre la doctrine selon laquelle le magistrat ne pouvait refuser son concours à la legis actio, c'est, me fautil répéter avec une expression qui n'a bien entendu dans ma pensée aucune acception désobligeante, en vertu d'une idée préconçue, parce qu'une pareille sujétion du magistrat lui apparaît comme une espèce d'inconvenance choquante, rationnellement inconcevable, comme une sorte d'impossibilité morale. ${ }^{1}$ Mais les impossibilités morales diffèrent des im-

en somme qu'on ne sait à quoi ils se rapportent. Oui peut-être et tout au plus, si on les prend séparément. Non, si on les rapproche; car alors on reconnait que le texte de Paul, extrait de son livre 56 $a d e d$. (Lenel, 692) où il traitait de la théorie de l'exécution sur la personne que les jurisconsultes étudiaient en partant du système de l'ancien droit (Lenel, Edictum ${ }^{2}, 1907$ ), commente le mot negare de la formule quando neque ais neque negas; et s'il le fait là, à propos de l'exécution, c'est que le défaut de négation autorisait l'exécution. Il me semble même que la scolie du Sinaï sur Ulpien, no 18 (P. Krüger, Collectio, 3, p. 281; Girard, Textes, p. 588) dans laquelle M. Mitteis a très finement découvert la preuve que le tacere du défendeur lui-même est uu lege agere, Z.S.St., 21, 1900, p. 103, s'accorde singulièrement bien avec notre doctrine sur le défaut de contradiction régulière et qu'elle confirme pleinement l'interprétation de Gaius, 2, 24, combattue par M. Wlassak.

1) Cette pensée intime apparait déjà au seuil du travail de M. Wlassak, à l'endroit où il soutient que le caractère des jours fastes résultait de paroles prononcées par le magistrat et non par les parties: il croit démontrer par là que le magistrat a le droit de refuser son concours à la legis actio, évidemment en ne concevant même pas, malgré le témoignage du Pro Murena (p. 131, n. 1), que le magistrat puisse être réduit à ce degré d'abaissement d'être obligé de répondre aux paroles des parties par d'autres paroles fixées d'avance. C'est du même état d'esprit que me parait procéder le passage $(28$, p. 98, n. 2) où, 
possibilités physiques en ce qu'elles ne sont pas les mêmes dans tous les temps ni dans tous les lieux. L'intérêt des études historiques bien conduites est précisément de nous familiariser avec ces diversités. M. Wlassak juge contraire à la dignité du magistrat romain d'avoir son activité commandée par les actes des parties en face desquels il derra prononcer des paroles fixées d'avance. Cependant, si tel est le rôle que lui assigne la procédure, ce n'est pas plus déshonorant pour lui que pour le receveur de l'enregistrement auquel on présente un acte à enregistrer et qui doit délivrer un certificat d'enregistrement sans s'occuper de la validité de l'acte, que pour le préfet qui reçoit la déclaration de candidature d'un personnage qu'il regarde comme un ennemi public et qui ne peut pas lui en refuser le récépissé. Le receveur enregistre l'acte; les tribunaux apprécieront s'il est valable. Le préfet délivre le récépissé; les électeurs voteront s'ils veulent contre le candidat. Le magistrat romain lie le procès; le juré le jugera. L'église catholique n'est pas moins jalouse de la dignité de ses prêtres que l'Etat romain de celle de ses magistrats. Cela n'a pas empêché que, pendant plus de trois siècles, depuis le concile de Trente jusqu'au décret ne temere du pape Pie $\mathrm{X}$ en date du 2 août 1907, le curé devant lequel deux de ses paroissiens arrivaient, même par surprise, à déclarer se prendre pour mari et

en sommant ses adversaires de lui répondre et en leur donnant luimême le programme de la preuve qu'ils auront à faire, il les invite à établir $1^{\circ}$ que leur doctrine s'appuie sur les sources; $2^{\circ}$ qu'elle peut se concilier avec le droit et l'histoire de la magistrature; $3^{\circ}$ que le régime qu'elle implique a pu fonctionner dans la vie et il conclut, sur le troisième point, par les mots: »ce que je conteste de la façon la plus énergique«. Heureusement pour nous, les contestations tirent leur valeur des arguments sur lesquels elles se fondent et non pas de l'énergie avec laquelle elles sont exprimées. Pour nous expliquer une fois sur ce programme en trois points, M. Wlassak remarque lui-même, quant au premier, que je lui opposerai sans doute le mécanisme des leges imperfectae et minus quam perfectae dont j'ai parlé p. 134; pour le second, si, comme je pense, il s'agit de la loi tirée du Strafrecht de Mommsen pour combattre les opinions de Mommsen, je me suis expliqué sur elle à la p.139; quant au troisième, les lignes qui suivent ont pour objet d'établir la possibilité pratique du système proclamé impraticable par M. Wlassak.

Zeitschrift für Rechtsgeschichte. XXIX. Rom. Abt. 
femme fut obligé de leur en décerner l'acte. Cela tenait à ce que, dans la notion du sacrement de mariage dont procédait cette règle, c'étaient les parties qui faisaient le sacrement et le prêtre jouait simplement le rôle de témoin nécessaire. ${ }^{1}$ ) La situation est toute pareille dans la legis actio. Elle est même en réalité encore plus simple, si on remarque que la legis actio est non seulement le préliminaire, mais la cause du jugement, crée un pouvoir de juger qui n'existe pas avant elle, que par conséquent personne n'a plus qualité pour connaitre de la demande avant l'achèvement de la legis actio que les tribunaux modernes n'ont qualité pour connaitre d'un procès avant l'exploit introductif d'instance. Se demander si, sous le système des legis actiones, le magistrat peut repousser la demande avant la legis actio, c'est se demander si un procès peut être perdu sans être intenté, si un procès peut être jugé avant qu'il y ait un juge. Si les choses ont changé par la suite, cela a été par une cause propre et par un phénomène en réalité très singulier, en vertu des pouvoirs nouveaux et véritablement exorbitants que le magistrat a tenus de la loi Aebutia.

Je n'ai rien de plus à dire sur la denegatio legis actionis: j'espère que, si d'autres me reprochent d'avoir été trop long, M. Wlassak ne pourra pas cette fois m'accuser d'avoir été trop bref. Mais ma défense n'est pas finie par là; car, si la sujétion du magistrat à la loi et en particulier son impuissance à arrêter une action légale dans la procédure antérieure à la loi Aebutia constituent un des principes fondamentaux desquels je suis parti, il est un peu exagéré de présenter, comme a fait $\mathrm{M}$. Wlassak dans la première partie de son travail publiée en $1904^{2}$ ), mon système sur la date de la loi Aebutia comme lié à cette idée au point de devoir vivre et mourir avec elle. En particulier en ce qui concerne la denegatio legis actionis, j'ai fait expressément remarquer, dans mon article de $1893^{3}$ ), que, si javais compris dans ma démonstration les textes relatifs à ce point, mon raisonne-

1) V. Esmein, Mariage en droit canonique, 2, 1891, pp. 182-183. Schulte, Lehrbuch d. katholischen Kirchenrechts ${ }^{3}$, 1873, p. 446, n. $15 .-$ 2) Z.S.St., 25, p. 131, n. 2. - $\left.{ }^{3}\right)$ Z.S.St., 14, pp. 39-40. N.R.H., 1897, pp. $279-280$. 
ment pouvait subsister à deux ou trois ans près, en éliminant ce terme et un autre relatif aux actions de bonne foi que je prévoyais pouvoir provoquer pareillement certaines résistances. Il resterait, par exemple, laction mandati in factum refusée contre les héritiers en 631 par le préteur urbain Sex. Julius, la formule mentionnée entre citoyens en 636 au plus tard par la loi latine de Bantia, la formule rutilienne et l'édit de Rutilius sur les operae en 636 au tard, l'édit de Drusus sur la iusiurandum liberti en 639 au plus tard, pour ne pas même descendre aux témoignages de la loi agraire de 643 sur la bonorum venditio et sur les clauses accessoires de la formule, exceptions ou prescriptions. C'est même, je suppose, pour cela que M. Partsch a indiqué ${ }^{1}$ ) ma démonstration comme plaçant la loi entre l'an 149 avant J. C. $=605$ de Rome et l'an $123=631$, tandis qu'il aurait indiqué comme second terme l'an $126=628$, s'il avait admis l'argument tiré de la denegatio legis actionis, et, malgré le langage de son premier article, M. Wlassak est parfaitement revenu dans le second sur mon système pour discuter, comme M. Mitteis et M. Cuq, certains de mes autres arguments.

Je crois en réalité pouvoir encore invoquer, après les cas de denegatio legis actionis, quatre ordres de faits comme établissant l'existence de la loi Aebutia avant laquelle ils ne pourraient se rencontrer. ${ }^{2}$ ) Ce sont en second lieu,

1) Schriftformel, p. 1, n. 3. - ${ }^{2}$ ) Si je n'avais le désir de limiter les répétitions, je pourrai même rationellement citer en sixième lieu, les édits, tels que ceux de Rutilius sur les operae (p.116, n. 4) et de Drusus sur la vindicatio in libertatem (p. 116, n. 6) qui, d'après Gaius, 4, 11 (p. 150, n. 1) sont comme les actions prétoriennes nécessairement postérieurs à l'introduction de la procédure formulaire. Je dois remarquer que l'un de mes contradicteurs $\mathbf{M}$. Cuq parait admettre ce criterium, 2, p. 732, n. 1 et $1^{2}$, p. 286, n. 3 . En effet, il invoque là, après M. Voigt, comme preure que la loi Aebutia aurait existé avant le VIIe siècle, la vetustas de l'édit prétorien attestée par Cicéron, De inventione, 2, 22,67. L'argument n'est pas pour me troubler beaucoup; car on cite tous les jours comme anciennes des choses qui ne datent pas de plus de einquante ou soixante ans, et il y a plus de soixante ans entre l'an 605 et l'époque où Cicéron, né nou pas en 646 , comme écrit M. Cuq, mais en 648 , a écrit le De inventione. Mais il ne peut être employé pour reculer la loi dans le passé que si on admet qu'il n'y avait pas d'édit prétorien avant elle. 
l'existence de formules entre citoyens qui nous est attestée dès l'an 636 au plus tard par la loi latine de Bantia; en troisième lieu, l'existence entre eux d'actions prétoriennes dont nous avons pour exemple l'action de mandat in factum refusée contre les héritiers par le préteur urbain Sex. Julius en 631 et la formule rutilienne créée par le préteur Rutilius en 636 au plus tard; en quatrième lieu, les actions de bonne foi dont Q. Mucius Scaevola, consul en 659, mort en 672, donne une si belle énumération, mais qui apparaissent au plus tard en 639 avec laction de mandat donnée contre les héritiers par le préteur Drusus; enfin en cinquième lieu, les exceptions qui, sous forme d'exceptions ou de prescriptions, surgissent avec l'édit de Rutilius sur les operae de 636 au plus tard et font l'objet d'allusions détaillées dans la loi agraire de 643. Nous pouvons nous dispenser de revenir sur les exemples puisqu'ils n'ont pas en général été contestés ${ }^{1}$ ); mais il nous faut d'autant plus insister sur les raisons par lesquelles on a pu vouloir écarter les criteriums.

2. J'admets que les exceptions ont existé seulement après la loi Aebutia, parce que Gaius dit dans un texte connu qu'elles n'étaient pas en usage au temps des Actions

1) Je n'aperçois guère de contestation à relever que pour l'édit de Drusus (p. 116, n. 6) au sujet duquel M. Wlassak remarque, 28, p. 55, n. 4, que ce Drusus pourrait être un autre des nombreux Livii qui ont occupé des magistratures; mais ce qui rend l'identification infiniment probable et ce qui a fait qu'elle a été adoptée à peu près unanimement (en dernier lieu par M. Mitteis, p. 140, n. 2), c'est le texte de la rhétorique à Herennius (p. 116, n. 2) qui dit du seul M. Livius Drusus, tr. pl. 632, cos. 642, qu'il a été préteur urbain. Pour trouver des contestations plus vivantes, il faudrait passer aux faits concrets que j'ai non pas invoqués, mais contestés en niant qu'ils prouvent l'existenco des contrats de bonne foi ou d'actions prétoriennes: la preuve de la vente vue dans Sex. Aeliưs à cause de $D ., 19,1,38,1$; celle de la vente et du louage vue dans Caton, De re rustica; celle de tous les contrats consensuels à peu près vue dans Plaute; celle de l'action rei uxoriae vue ailleurs encore. Mais, sauf les observations que je ferai plus loin sur une conception où ces textes prouveraient l'existence des actions qu'on y voit, non pas après la loi Aebutia, mais avant elle (p. 154 et ss.), je ne vois rien à retirer des explications que j'ai déjà données Z.S.St., 14,1893 , pp. $21-23$, et plus complètement pour l'action rei uxoriae N.R.H., 1897, pp. 259-261. 
de la loi. $\left.{ }^{1}\right)$ M. Wlassak répond que le texte ne dit pas cela; qu'il dit seulement et qu'il dit littéralement que les exceptions n'étaient pas employées sous les Actions de la loi de la même façon que du temps de Gaius. ${ }^{2}$ )

C'est une façon d'écarter le texte de Gaius qui m'est bien connue comme sans doute à quelques autres hommes de mon âge. $D u$ temps où j'étais étudiant, elle tenait sa place dans l'arsenal d'expédients à l'aide desquels les maîtres d'une école qui eut son heure d'influence en Allemagne et qui survécut plus longtemps encore en France, effaçaient méthodiquement, sous prétexte de sens commun, dans l'ancienne procédure romaine, tous les traits originaux qui l'auraient mise en conflit trop choquant avec l'état d'esprit des praticiens modernes. Si même j'étais enclin aux effusions mélancoliques, $\mathrm{j}$ 'exprimerai bien sincèrement ma tristesse de voir cet argument de l'ancienne école $d u$ bon sens repris par un romaniste qui, en d'autres points, a si brillamment employé les méthodes modernes. Je me bornerai à dire que cette prétendue interprétation littérale est, peut-on démontrer, sûrement fausse.

Il n'est même pas besoin de revenir sur les explications que j'ai données au sujet de la denegatio legis actionis. Elles ne permettraient pas, je crois, que le texte signifiat, comme le veut M. Wlassak, que l'exception était sous les Actions de la loi examinée avant la litis contestatio, pour amener le magistrat à refuser la legis actio, au lieu de l'être après, pour amener le juge à repousser la demande. Mais l'impossibilité de la traduction ressuscitée par M. Wlassak résulte du texte même de Gaius. Il pourrait à la rigueur vouloir

1) Gaius, 4, 106: Et si quidem imperio continenti iudicio actum fuerit ... postea nihilominus ipso iure de eadem re agi potest; et ideo necessaria est exceptio rei iudicatae vel in iudicium deductae. 107. Si vero legitimo iudicio in personam actum sit ea formula quae iuris civilis habet intentionem, postea ipso iure de eadem re agi non potest et ob id exceptio supervacua est: si vero vel in rem vel in factum actum fuerit, ipso iure nihilominus postea agi potest, et ob id exceptio necessaria est rei iudicatae vel in iudicium deductae. 108. Alia causa fuit olim legis actionum, nam qua de re actum semel erat, de ea postea ipso iure agi non poterat; nec omnino ita ut nunc usus erat illis temporibus exceptionum. - 2) Wlassak, 28, p. 100 à la note. 
dire que l'exception ne s'invoquait pas sous les Actions de la loi de la même façon que sous la procédure formulaire s'il se trouvait dans un exposé général du système des exceptions. Seulement cela n'est pas. La phrase appartient à un développement d'ordre tout différent. Elle figure incidemment dans un développement sur l'effet extinctif de la litis contestatio. Gaius dit d'abord, au sujet de la procédure formulaire, dans quels cas l'action subsiste ipso iure et par suite on a besoin de l'exception rei iudicatae vel in iudicium deductae et dans quels cas le droit d'agir disparait ipso iure et par suite on n'a pas besoin d'exception, en visant évidemment la distinction classique des défenses ipso iure invoquées devant le juge sur l'intentio même de la formule et des exceptions qui doivent lui être soumises par une clause spéciale. Puis il passe au temps des Actions de la loi et il dit qu'alors le droit d'agir disparaissait toujours ipso iure et il n'y avait pas encore d'exception, en employant naturellement les mêmes expressions dans le même sens, en ne les employant pas dans le sens supposé par M. Wlassak qui serait tout différent, et où il faudrait de plus, remarquons-le, que le terme ipso iure fut pris dans une acception tout à fait anormale, avec la signification d'imperio praetoris.

3. J'ai admis que les actions prétoriennes ont existé seulement après la loi Aebutia, parce qu'un autre texte connu de Gaius dit qu'au temps des Actions de la loi les édits prétoriens qui ont introduit plusieurs actions n'étaient pas encore en usage. ${ }^{1}$ )

M. Wlassak oppose encore une traduction différente venant de lui, qui est beaucoup plus nouvelle que la précédente et qui a d'ailleurs été immédiatement adoptée par $\mathbf{M}$. Cuq, par M. Mitteis ${ }^{2}$ ) et par quelques autres auteurs désireux

1) Gaius, 4, 11: Actiones, quas in usu veteres habuerunt, legis actiones appellabantur vel ideo, quod legibus proditae erant (quippe trone edicta praetoris, quibus conplures actiones introductae sunt nondum in usu habebantuir), vel ideo quia ipsarum legum verbis accomodatae erant et ideo inmutabiles proinde atque leges observabantur: - 2) Wlassak, Proce $\beta$ gesetze, 2, p. 303 et ss. Zur Geschichte der Cognitur, 1893, p. 59 et ss. Cuq, Institutions, 2, p. 732, n. 4. Mitteis, Römisches Privatrecht, pp. 42, n. 5. 49. 51, n. 43. M. Mitteis ajoute, p. 49, comme argument de fond, que l'existence des interdits avant la loi Aebutia prouve que le pré- 
de placer la création d'actions prétoriennes particulières à des dates où la loi Aebutia leur paraissait ne pouvoir pas encore avoir été rendue.

Suivant M. Wlassak, Gaius ne voudrait pas dire, comme on avait toujours pensé, que les édits prétoriens ${ }^{1}$ ) qui ont introduit plusieurs actions n'étaient pas encore en usage au temps des Actions de la loi, mais qu'ils n'étaient pas encore en usage au temps où les Actions de la loi ont été introduites, - ce qui rapporterait le témoignage sur l'inexistence des actions prétoriennes non pas à toute la période de la domination des Actions de la loi, mais uniquement à l'époque de l'introduction de cette procédure.

Ce n'est assurément pas la traduction la plus naturelle: la preuve en est que personne ne s'en était avisé avant que M. Wlassak l'imaginât pour les besoins de son système. Ce n'est pas non plus, je crois, celle qui attribue à Gaius la façon la plus heureuse de s'exprimer. Mais ce qu'il faut relever surtout, e'est que même si elle était admise, cette

teur a pu dès lors créer des actions. Mais c'est, à mon sens, confondre deux domaines très différents. Le préteur ne peut ni donner une action que la loi ne donne pas, ni refuser une action que la loi donne. Mais en dehors de là, à condition de ne pas franchir cette double limite, il est libre de rendre en vertu de ses pouvoirs généraux de magistrat, des injonctions et des défenses qu'il fera respecter par ses moyens d'autorité de droit commun: ce qui n'exclut ni les missiones in possessionem, ni les stipulations prétoriennes, ni les interdits euxmêmes à condition qu'il les rende purement et simplement en les faisant respecter multis et pignoribus ou même conditionnellement en forçant ensuite par le même moyen les parties à faire les stipulations de la procédure per sponsiones. Cf. Organisation judiciaire, 1, pp. 203206. Manuel, pp. 989-990: trad. pp. 1085-1086. Tout cela n'est pas prouvé; mais rien de plus n'est prouvé, et rien de tout cela ne contrarie notre principe. On peut même remarquer, à la façon dont les interdits possessoires se glissent dans un des rares interstices du droit procédural où la liberté du magistrat n'était pas supprimée, dans le domaine de l'attribution des vindiciae, combien on savait à la fois respecter scrupuleusement la barrière et en utiliser ingénieusement les moindres solutions de continuité.

1) Je crois présentement superflu pour cette question de discuter ici les conclusions qui ont été tirées par M. Wlassak du singulier: praetoris (remplacé par P. Krüger, Gesch. der Quellen, 1888, p. 37, n. 29, par le pluriel: praetorum) dans Gaius, 4, 11 (p. 150, n. 1). 
traduction n'aboutirait pas du tout au résultat poursuivi par M. Wassak. Ce que le texte signifierait, c'est que les actions prétoriennes encore inconnues au temps des lois qui ont introduit les différentes Actions de la loi ont apparu après les lois, toutes les lois qui ont créé les Actions de la loi, non pas seulement après la loi des XII tables, mais après la loi Silia et la loi Calpurnia sur la condictio, après les lois de la première et de la seconde moitié du VI ${ }^{\mathrm{e}}$ siècle, qui ont créé des manus iniectiones pro iudicato et des manus iniectiones purae. L'interprétation contournée, dont je ne suis au reste pas plus partisan ici, pour le texte de Gaius relatif aux actions prétoriennes, que tout à l'heure, pour son texte relatif aux exceptions, aboutirait sensiblement au même résultat que l'interprétation naturelle. C'est une raison de plus de préférer l'interprétation naturelle.

4. J'estime encore que les actions de bonne foi n'ont existé que depuis l'introduction de la procédure formulaire par la loi Aebutia: car Cicéron dit, dans un passage du $D e$ officiis, qu'elles ont été établies sine lege "), et Gaius dit, c'est sa première explication du nom des Actions de la loi, qu'elles n'existaient qu'en vertu de lois positives. Il est vrai que des interprètes ont objecté que Cicéron ne voulait peut-être pas dire cela, qu'il voulait peut-être simplement dire que la clause ex fide bona qui y réprime le dol y était insérée sine lege. Mais il a depuis longtemps été répondu que, si l'on traduit ainsi, les actions de bonne foi n'en restent pas moins exclues du système des Actions de la loi par le texte de Cicéron, non plus en vertu de la première explication du nom des Actions de la loi donnée par Gaius, mais en vertu de la seconde, parce qu'elles reproduisaient les termes mêmes de la loi sans pouvoir rien y ajouter. Par conséquent, que Cicéron veuille dire que les actions de bonne foi ont été établies sine lege ou qu'elles contiennent leur clause caractéristique sine lege, son texte prouve qu'elles n'existaient pas sous les Actions de la loi, et on peut con-

1) De off., 3, 15, 61: Atqui iste dolus malus et legibus erat vindicatus, ut in (in manque dans les mss.) tutela duodecim tabulis, circumscriptio adulescentium lege Plaetoria, et sine lege iudiciis, in quibus additur ex fide bona. 
sidérer l'apparition des unes ou des autres d'entre elles comme un signe de l'existence de la loi Aebutia.

5. Enfin nous croyons que les formules n'ont été employées entre citoyens que depuis la loi Aebutia; car Gaius dit expressément, en employant une tournure possessive dont M. Wlassak lui-même souligne le sens bien connu, que ce sont la loi Aebutia et les lois Juliae qui ont fait que les citoyens ont plaidé par formules. ${ }^{1}$ ) Et c'est pourquoi $\mathbf{M}$. Kniep a fourni une nouvelle preuve de l'existence de la loi Aebutia $^{2}$ ) en relevant la mention explicite de la formule faite en l'an 636 au plus tard par la loi latine de Bantia ${ }^{3}$ ) au sujet d'un procès qui est nécessairement un procès entre citoyens, puisque l'action dont il s'agit ne peut être intentée que par un magistrat et contre un magistrat ou un sénateur,

1) Gaius, 4, 30 ... ut per concepta verba id est per formulas litigemus. Cf. Wlassak, Proceßgesetze, 2, p. 184, n. 2. C'est à tort que M. Cuq, dans Darenberg et Saglio, p. 1127, a présenté notre système comme impliquant qu'il n'y aurait pas eu de formules pour les pérégrins avant la loi Aebutia. V. plus haut, p. 122, n. 1. - 2) Societas publicanorum, 1, 1896, pp. 444-445, où il remarque que Bruns, Z.R.G., 3, 269, et Huschke, Multa, p. 262, paraissent bien avoir déjà entendu le texte d'une formule. - ${ }^{3}$ ) C.I.L., I, 197. Bruns, Fontes ${ }^{6}$, pp. 53-55. Girard, Textes ${ }^{3}$, pp. $29-31$. Cf. plus haut, p. 117, n. 6. L'argument ne disparaitrait que dans la doctrine émise tout récemment par $M$. Maschke, Theorie und Geschichte der römischen Agrargesetze, 1906, selon laquelle la loi latine de Bantia, placée unanimement entre l'an 621 et l'an 636, à raison de la mention qu' y a relevée Mommsen des seuls triumvirs agraires qui aient été organisés comme magistrats ordinaires annaux, c'est-à-dire des triumvirs créés par la loi agraire de Ti. Gracchus et abolis par la loi Thoria de 635 ou 636 , serait au contraire un fragment de la loi agraire Appuleia de l'an 654. M. Maschke, qui s'appuie sur le caractère de loi jurée par les magistrats et les sénateurs que l'on sait avoir appartenu à la loi Appuleia comme à la loi de Bantia, soutient que les $I I I$ viri de la loi de Bantia pourrajent être, aussi bien que les triumvirs ordinaires annaux de la loi Sempronia, de simples triumvirs extraordinaires, ceux créés par la loi Appuleia elle-même; car, dit-il, le seul passage de la loi qui serait incompatible arec cette idée, celui d'après lequel ils devraient prêter serment à la loi dans les cinq jours où ils en auraient connaissance, est le produit d'une restitution. Mais cette restitution n'a été faite qu'en partant de la partie conservée et cette partie conservée elle-même ne peut s'entendre que de triumvirs ayant le caractère de magistrats ordinaires comme les magistrats avec lesquels ils sont énumérés. 
qu'elle est donnée à tous les magistrats contre les magistrats ou sénateurs coupables de violation de la loi.

A la vérité, les auteurs qui ont essayé d'écarter le témoignage de Gaius pour soutenir qu'il y avait des actions prétoriennes avant la loi Aebutia ont traité le texte de Gaius sur les formules et celui de Cicéron sur les actions de bonne foi comme s'ils n'existaient pas en soutenant, sans entreprendre la discussion du dernier ni reprendre celle du précédent, que les formules et les actions de bonne foi auraient déjà été pratiquées par les citoyens sous les Actions de la loi dans une forme qui les confondrait plus ou moins avec les actions prétoriennes. ${ }^{1}$ ) Ce serait au moyen des arbitria

1) Mitteis, pp. 46-53. Cuq, 1, 1891, pp. 690-694. 2, 1902, pp. 733, n. 4. $1^{2}, 1904$, pp. $169.225 .285,5.286,6$. M. Wlassak reste plus réservé sans avoir peut-être des idées très différentes. La doctrine avait déjà été exprimée par M. Karlowa, Civilprozeß, 1872, pp. 140-252 et par Hertmann-Ubbelohde, Ordo iudiciorum, 1, 1886, p. 271 et ss. Mais M. Mitteis reconnait, pour le dernier ourrage, qu'il donne ses arguments sans en faire le triage et note, pour le premier, qu'il repousse résolument l'idée d'un programme analogue à la formule. M. Cuq lui-même a d'ailleurs varié sur ce point, remarque $M$. Mitteis au même lieu, et, comme mon observation se rapporte à des critiques dirigées par M. Cuq contre moi, j'ai le droit de faire observer qu'il ne parait pas encore être arrivé à des solutions parfaitement concordantes. En effet, tandis que d'après cette doctrine il y a eu des formules et (sauf explication terninologique) des contrats de bonne foi avant la loi Aebutia comme après, il soutient que la loi Aebutia doit être du VIe siècle et non du VII' non seulement par l'argument tiré de la vetustas de l'édit, 2, 732, 1. $1^{2}, 286,5$, déjà signalé p. 147, n. 2, mais à cause du grand nombre de formules qui existaient au temps où Cicéron écrivit les Topiques, d'après Top., 8, 33 cité, 2, p. 732, n. 1 et à celui où il prononça le Pro Roscio com. d'après Pro Roscio 8, 24 cité $1^{2}$, p. 286, n. 1, et, dit le tome 2, p. 732, n. 1, à raison des divers textes qui prouvent l'existence de l'arbitrium en matière de vente dans la seconde moitié du VI ${ }^{\theta}$ siècle et sa transformation en iudicium en vertu d'un accord préalable entre les parties (M. Cuq renvoie au texte sur Sextus Aelius, $D ., 19,1,38,1$, et à Caton, De re rustica, 149). Il ne m'est pas très malaisé de répondre, pour les formules, que, le préteur ayant été à mon sens chargé par la loi Aebutia de transformer toutes les legis actiones en formules et en ayant tiré le pouvoir de donner des formules dans des cas où il n'y avait pas de legis actio, il a dû en effet rédiger beaucoup de formules non seulement dans l'espace de plus d'un siècle qui sépare l'an 605 de l'an 710 où furent écrits les Topiques, mais 
honoraria dont on croit trouver des vestiges plus ou moins nombreux à une époque antérieure à l'introduction légale de la procédure formulaire, M. Mitteis dans les formulaires du traité d'économie rurale de Caton et dans un texte de Celse rapportant par le canal de Drusus une décision de Sex. Aelius, M. Cuq en outre dans Plaute. J'ai dit depuis longtemps et plus d'une fois ${ }^{1}$ ) pourquoi ces textes ne contiennent pas ce qu'on y voit. Mais s'ils le contenaient, s'ils

dans celui de plus de soixante dix ans qui sépare cette année de celle où il plaida pour Roscius; pour les contrats de bonne foi, que j'ai déjà dit pourquoi ces textes ne me paraissent pas établir la reconnaissance des contrats de vente et de louage. Et même je peux noter que, comme je me suis expliqué p. 153, n. 1, sur l'objection contenue dans Daremberg et Saglio et p. 147, n. 2, sur celle tirée de la vetustas edicti, j'ai répondu à toutes les objections propres de $\mathrm{M}$. Cuq. Mais ce que je dois souligner, c'est que les deux objections que M. Voigt a pu m'adresser parce qu'il considérait qu'il n'y avait ni formules ni actions de vente et de louage avant la loi Aebutia ne devraient pas logiquement m'être opposées par M. Cuq; car, quand on admet qu'il y avait des formules, en matière de vente et de louage comme ailleurs, avant la loi Aebutia comme après, on ne peut pas invoquer, comme preuve de l'existence de la loi Aebutia, l'abondance des formules ni la reconnaissance des contrats de vente et de louage.

1) V. p. 148, n. 1. Je ne crois pas avoir à discuter ici la conjecture hardie de M. Segré, Sulle formule relative alla neg. gestio, 1905, p. 25 et ss. adoptée dubitativement par M. Mitteis, p. 57, n. 45, selon laquelle le iusiurandum de operis, sanctionné par la même action civile que la stipulation operarum, aurait été auparavant sanctionné par une action prétorienne qui serait déjà née, à un âge assurément bien reculé, du serment, ni la conjecture pareillement hardie de M. Mitteis, p. 53, n.31, selon laquelle la cautio rei uxoriae d'Aulu-Gelle, 4, 3, 1, que M. Mitteis suppose avoir d'abord été surtout pratiquée dans des mariages avec manus où la conventio in manum aurait éteint toute créance civile de la femme contre le mari, aurait été un simple pacte tendant à la nomination éventuelle d'un arbitre. Car ce sont là, je pense, plutôt des hypothèses qui pourraient s'appuyer sur le système démontré que des arguments pouvant servir à appuyer le système. Liidée de M. Mitteis p. 51, n. 28, selon laquelle l'opposition des iurgia et des lites pourrait être rattachée à la distinction de ces procès honoraires et des procès civils, me parait contrariée par l'observation que les exemples les plus sûrs de iurgia sont les actions finium regundorum et familiae erciscundae (Cicéron et Nonius dans Bruns, 2, p. 66; Papinien, $D$., 10, 2,57) qui s'intentent par legis actio en vertu de la loi des XII tables (Cicéron, De leg., 1, 21; D., 10, 2, 1, pr.). 
attestaient l'existence d'actions sanctionnant le contrat de vente ou le contrat de louage avant la mort de Caton mort en 605, au temps de Sex. Aelius consul en 556, avant la mort de Plaute mort en 570, ce qu'ils prouveraient ce serait l'existence de la loi Aebutia, ce ne serait pas celle des prétendus arbitria honoraria en discordance manifeste avec le triple principe d'exclusion des formules, des actions prétoriennes et des exceptions avant la loi Aebutia. Tout en admettant l'existence de ces arbitria, M. Mitteis a signalé le caractère inexact et contradictoire d'une partie des arguments présentés en leur faveur. $M$. Cuq, qui est l'auteur récent qui a tenté d'en exposer le plus explicitement le système, s'est trouvé montrer par là du même coup la disproportion formidable qui existe entre la hardiesse de l'édifice et la fragilité de ses fondations. $\Pi$ dit, en un lieu en renvoyant pour la preuve à un autre, que ces arbitria distincts de l'arbitrage extrajudiciaire rendu obligatoire par contrat verbal intervenaient entre citoyens quand il s'agissait de prétentions qui n'étaient pas sanctionnées par la loi, qui étaient seulement obligatoires d'après l'usage des honnêtes gens, comme par exemple celles résultant de la tutelle, de la société, de la fiducie, de la vente, du louage, du mandat, en somme des principaux futurs contrats et quasi-contrats de bonne foi, et que les parties obtenaient du préteur de délivrer une formule grâce à laquelle la décision de l'arbitre avait la même valeur que la sentence d'un juge. ${ }^{1}$ ) Puis, à l'en-

1) Institutions, 12, p. 225: "Les parties peurent la rendre (la sentence arbitrale) juridiquement obligatoire de deux manières: $1^{0}$ par un compromis ...2 $2^{\circ}$ en demandant au préteur de conférer à l'arbitre le pouvoir d'un juge. Si la demande est agréée (M. Cuq cite en note le texte de la Rhétorique à Herennius, p. 116, n. 2, relatif au mandat et un texte de Paul en matière de gestion d'affaires qui parlent tous deux de iudicium), le magistrat délivre aux parties une instruction écrite qui précise la cause du débat et autorise le juge à absoudre le défendeur ou à le condamner dans la mesure qui lui paraitra équitable. L'emploi de ce second moyen, dès le VIe siècle a été contesté; mais nous établirons (p. 285, 4) que l'application de la procédure formulaire aux procès entre citoyens se rattache à la convention d'arbitrage. Cette procédure a été généralisée, et non créée par la loi Aebutia et par les lois judiciaires d'Auguste.« V. auparavant, p. 223, la liste des opérations visées. 
droit indiqué pour la preuve ${ }^{1}$ ), il signale le rapport entre la convention d'arbitrage et la procédure formulaire comme attesté par deux faits: l'accord des volontés de parties qui serait également requis pour le renvoi de l'affaire à un juge ou à un arbitre et la manière dont Cicéron appellerait les actions précitées tantôt arbitria ex bona fide, tantôt arbitria honoraria et tantôt iudicia sine lege. Il y aurait beaucoup à dire sur tout cela ${ }^{2}$ ) et en particulier sur les deux derniers

1) Institutions, $1^{2}$, p. 285: »L'usage de la procédure formulaire parait avoir été étendu aux procès entre citoyens, dans des cas où les actions de la loi n'étaient pas applicables (4). Le préteur urbain délivrait une formule sur la demande des parties, lorsque étant d'accord pour faire trancher leur différend par voie d'arbitrage, elles voulaient que la sentence de l'arbitre eût la même valeur que celle d'un juge (5).« La note 4 contient un renvoi à Lenel, Z.S.St., 24, 329, c'est à dire à l'article sur la forme de la litis contestatio, pp. 329-343 où M. Cuq vise sans doute les idées sur l'origine arbitrale de la Justice exprimées pp. 342-343. La note 5 porte que »le rapport entre la procédure formulaire et la convention d'arbitrage est attesté « par les deux faits signalés au texte. V. sur eux la p. 158, n.1. - ${ }^{2}$ ) L'histoire des arbitria et des arbitri nous parait se dessiner de la manière la plus naturelle et la plus conforme aux sources si l'on considère le nom comme ayant été d'abord donné conformément à l'étymologie à ceux des juges saisis de par legis actio qui statuaient après un examen des lieux (actions familiae crciscundae, finium regundorum, aquae pluriae arcendae, arbitrium liti aestimandae: Eisele, Beiträge, 1896, pp. 1-15; Organisation judiciaire, 1, pp. 82, n. 2. 94, n. 4) et comme s'étant étendu de là soit aux actions créées par la procédure formulaire où le juge avait un pouvoir d'appréciation également large (Cicéron, De Off., 3, 17, 70. Pro Roscio, 4, 10; Sénèque, De Benef., 3, 7), soit à l'arbitrage extrajudiciaire dont le rôle a été beaucoup plus effacé dans l'ancienne Rome que dans la plupart des sociétés primitives, soit à tous les cas où une personne était chargée d'une appréciation quelconque. Pour admettre en outre les arbitria honoraria qui auraient, d'après la doctrine nouvelle, fonctionné avant la loi Aebutia ì côté des legis actiones, il faudrait supposer nou seulement que les personnes qui concluaient des arrangements non obligatoires avaient l'habitude de s'entendre pour soumettre à des arbitres leurs scrupules de conscience relatifs à ces arrangements, mais que le préteur, chargé par la loi de donner des juges seulement pour les procès liés par legis actio, aurait consenti à en donner en vertu de ces conventions d'arbitrage quand on le lui aurait demandé; puis qu'il aurait consenti à leur donner des instructions semblables à la future formule; puis qu'il aurait voulu et pu donner à la sentence rendue par ces arbitres sans legis actio le même effet qu'à celle rendue sur 
points. ${ }^{1}$ ) Mais ils seraient tous deux de la plus entière exactitude qu'ils ne fourniraient pas la moindre preuve que le préteur ait là plus qu'ailleurs délivré des formules entre citoyens avant la loi Aebutia.

une legis actio; enfin, pour que le système prit un certain développement, que le défendeur ait pu être contraint, en cas de résistance, à accepter l'arbitrage. A notre avis, aucune de ces suppositions n'est appuyée sur des faits et la plupart sont condamnées par les textes les plus sûrs.

1) Pour le second point le texte de Cicéron, 3, 15, 61, qui ne parle pas, comme écrit M. Cuq, de »iudicia sine lege« ainsi appelés »parce que ce sont des actions qui n'ont pas été réglées par la loi, qui ne donnent pas lieu à une action de la loi«, qui dit seulement que le dol était réprimé lege en matière de tutelle par la loi des XII tables et de circumscriptio minosum par la loi Plaetoria et sine lege judiciis quibus additur ex fide bona, ne souffle mot, ni dans une traduction, ni dans l'autre, de l'existence des arbitria honoraria avant la loi Aebutia. Celui de Cicéron, De off., 3,17, 70, dans lequel non plus il n'appelle pas précisément les actions de la vente et du louage arbitria ex fide bona, mais il dit que Mucius Scaevola summam vim esse dicebat in omnibus arbitriis in quibus adderetur ex fide bona et pensait que cela s'appliquait dans les actions de vente, de louage, etc. parle encore des actions de bonne foi in quibus additur ex fide bona en employant simplement, comme il arrive souvent pour elles, au lieu du terme iudicia le terme arbitria, parce que le juge y avait les mêmes pouvoirs larges que dans les anciens arbitria intentés par legis actio; mais il ne dit en rien que ces arbitria, qui sont des iudicia, qu'il appelle de ce nom, aient été créés par le préteur, encore moins qu'ils l'aient été par lui sous les Actions de la loi. Enfin le seul des textes invoqués qui parle d'arbitria honoraria (M. Cuq omet avec raison deux passages des Tuscul., 5, 41, 120, et du De fato, 17, 39, qui parlent d'arbiter honorarius en se rapportant au rôle honorable de l'arbiter ex compromisso) celui du Pro Rosc. com., 5, 15, qui cite les arbitria honoraria après les iudicia legitima et avant les officia domestica, ne peut, a montré M. Mitteis, p. 47, n. 1, s'entendre d'une opposition avec les procès civils des procès honoraires qui, au temps de Cicéron, seraient eux-mêmes devenus des iudicia legitima, en vertu de la loi Aebutia; c'est sans doute, comme il a pensé, un exemple de phraséologie incorrecte, mélangeant l'opposition des moyens prétoriens et civils et celle des actions d'interprétation large ou rigoureuse. - Sur le premier point, il faudrait pour que le rapprochement fut exact que le défendeur fut contraint d'accepter l'arbitrage comme il l'est de consentir au procès. M. Cuq semble avoir tenté de le démontrer en écrivant: »Lorsqu'on n'avait pas conclu d'avance la convention d'arbitrage, on n'était pas libre de décliner l'arbitrage d'un homme de bien. Le 
IV.

Voilà le résultat de mon enquête sur la position actuelle du problème que j'avais déjà abordé en 1893. Pas de documents nouveaux d'une portée réelle. Quant aux matériaux anciens, j'étais parti des principes qui me paraissaient les mieux établis sur l'histoire des institutions procédurales de Rome pour chercher les faits qui, en vertu de ces principes, attesteraient l'existence à une date ou à une

demandeur peut arbitrum adigere (Cic., De off., 3, 16; Top., 10). Adigere implique l'idée d'une contrainte morale (cf. Iusiurandum adigere: Liv. XLIII, 7; XXXVIII, 39). La sanction consistait dans le blâme du censeur (Ascon., 84, Orelli)«. Mais les textes cités ne prouvent rien de pareil. D'abord le terme adigere arbitrum, qui impliquerait une obligation pour l'arbitre d'arbitrer plutôt qu'une obligation de l'autre partie de consentir à l'arbitrage, se rapporte, dans Top., 10, 43, aux actions finium regundorum et aquae pluviae arcendae qui s'intentaient par legis actio en vertu des XII tables (D., 10, 1, 13; 41, 7, 21, pr.) et dans $D e$ off., $3,16,66$, à une action empti soumise au père de Caton d'Utique né au plus tôt vers 620 (Manuel, 530, trad. 585) non pas dans Ia forme conjecturale qu'elle aurait eue au temps des arbitria honoraria, mais dans la forme connue de la procédure formulaire avec l'intentio: quidquid dare facere oportet ex fide bona; il ne prouverait par conséquent l'existence d'une contrainte quelconque que dans des procès intentés par legis actio, sous les Actions de la loi, ou par formules, sous la procédure formulaire, et pas du tout pour les conventions déférées à l'arbitrage sans être protégées par la loi avant la loi Aebutia. Quant à la contrainte éventuelle et à échéance lointaine résultant de la perspective de la nota des censeurs, elle serait tout autre chose que la contrainte immédiate à accepter le procès produite, dans notre doctrine, par l'assimilation de celui qui ne se défend pas au iudicatus ou, dans celle de M. Wlassak, par la missio in possessionem prononcée contre lui. Mais le texte d'Asconius ed. Orelli, p. 34, ed. Kiessling, p. 75, ne la prouve pas pour une prétention non reconnue par la loi, pour laquelle une personne aurait refusé d'accepter l'arbitrium honorarium avant la loi Aebutia; car l'affaire à laquelle il se rapporte est une affaire de la fin du VIIe siècle, l'histoire du consul de l'an 691 C. Antonius qui, ayant été poursuivi en l'an 678 devant le préteur pérégrin Lucullus par des Grecs qu'il avait spoliés, empêcha le renvoi du procès aux jurés en demandant l'intercession des tribuns sous prétexte qu'il n'aurait pu plaider à armes égales contre ces étrangers à Rome (cf. Cicéron, Or. int. tog. cand. 2; Q. Cicéron, Pet. cons. 8) et qui fut en conséquence exclu du sénat par les censeurs de 684, en même temps que pour d'autres méfaits, quod iudicium recusarit. 
autre de l'innovation opérée par la loi Aebutia. Il se trouve que ceux qui ont repoussé mes conclusions n'ont pour ainsi dire pas contesté les faits, que leurs critiques ont porté presque exclusivement sur les principes que j'avais pris pour base et qui sont les principes mêmes qui ont été dégagés, en face des erreurs anciennes, par les maîtres qui ont le plus contribué au progrès de la science du droit romain, par ceux qui ont le plus efficacement appliqué à son étude les méthodes scientifiques modernes. Il résulte de là que ma réfutation, si elle était faite, impliquerait beaucoup plus que la chute d'un système émis par une personne sur la date d'une loi de procédure. Elle impliquerait la disparition d'une partie des vues historiques fondamentales qui apparaissaient comme un des profits les plus précieux dus aux recherches modernes sur l'ancienne procédure. Mais il me semble que mon travail de révision montre que ce danger n'est pas trop à craindre.

Pour défendre la conception qui donne à la procédure romaine la physionomie la plus conforme, non pas à ces vraisemblances superficielles qui confondent les âges et les lieux, mais aux vraisemblances scientifiques tirées de la similitude des institutions dans les groupes du même type, la conception selon laquelle la procédure judiciaire, en sortant de la procédure extrajudiciaire, a conservé les mêmes traits originaux et archaïques à Rome qu'ailleurs, la conception suivant laquelle, à Rome comme ailleurs, le simple a précédé le compliqué et les distinctions d'actions rigoureuses et d'actions d'interprétation libre, d'actions données par la loi et d'actions données sans loi n'ont pas été un phénomène pour ainsi dire primitif, mais le produit d'une juxtaposition relativement récente, je ne crois pas avoir eu besoin de faire violence ni aux textes ni aux faits. Je n'ai eu qu'à prendre les textes dans leur sens le plus naturel, sans avoir nulle part à remplacer les traductions qui viennent les premières à l'esprit et qui sont le plus souvent les meilleures, par ces interprétations forcées qui sont toujours suspectes et dont l'invraisemblance croit à l'infini à mesure qu'il faut les multipier, sans avoir non plus jamais à recourir à des expédients désespérés tels par exemple que celui que $\mathbf{M}$. Wlassak 
annonce quelque part avoir l'intention d'employer en faveur d'une de ses doctrines en soutenant que »la plupart des formules (conservées dans le manuscrit de Vérone des Institutes de Gaius) ont déjà été falsifiées par les auteurs du palimpseste ${ }^{1}{ }^{1}$ ) Cela donne à croire que les idées historiques que j'avais prises comme instrument de recherches chez les savants les plus éclairés ne seront pas si facilement écartées. C'est un des caractères des vérités scientifiques une fois découvertes de ne pouvoir plus ensuite être masquées d'une manière durable. Si je ne l'ai pas assez bien montré pour celles-là, c'est ma faute, non la leur et le moment ne se fera pas longtemps attendre où un autre les défendra mieux.

Pour la loi Aebutia, je finirai en soumettant au lecteur deux considérations générales qui me semblent fournir un solide appui aux conclusions que j'ai obtenues par des procédés indépendants. Selon M. Wlassak, M. Mitteis, M. Cuq et sans doute encore d'autres partisans des mêmes doctrines qui ne les ont pas exprimées par écrit, il y a eu, avant la loi Aebutia comme après, aussi bien pour les citoyens que pour les étrangers, des formules, des actions de bonne foi, des actions prétoriennes, des exceptions, des magistrats en droit d'accorder ou de refuser des actions en dehors de la loi. C'est entendu. Mais alors comment concevoir la portée de la loi, d'une part? Comment, d'autre part, expliquer la différence de physionomie juridique qu'il est impossible de méconnaître entre les périodes antérieure et postérieure aux premiers temps du VII ${ }^{\mathrm{c}}$ siècle de Rome, à la fois dans le champ procédural proprement dit et dans les domaines limitrophes?

D'abord quelle a été la portée de la loi? On ne peut nier qu'elle se réduit à bien peu de chose, si la loi n'a fait que sanctionner ce qui existait, que reconnaître au magistrat des pouvoirs qu'il exerçait déjà. C'est une conséquence forcée de la doctrine selon laquelle les formules et tout le reste existaient avant la loi Aebutia comme après. Seulement c'est en désaccord absolu avec les deux seuls textes qui parlent expressément de la loi: le texte d'Aulu-Gelle

1) Z.S.St., 25, p. 139. 
qui dit qu'elle a eu pour suite la disparition de certaines antiquités des XII tables telles que les vades et les subvades, la perquisition lance licioque et les peines du talion et de vingt-cinq as du délit civil d'injures ${ }^{1}$ ) et le texte de Gaius selon lequel la loi Aebutia et les lois Juliae ont mis les formules à la place des legis actiones. ${ }^{2}$ ) Ces textes se comprennent si, comme nous le croyons, la loi Aebutia a introduit les formules et tout ce qui en a dérivé, tandis que les lois Juliae ont définitivement aboli les legis actiones. Alors Gaius peut présenter dans un raccourci la réforme d'ensemble qui a substitué les formules aux Actions de la loi comme accomplie par la loi Aebutia qui a introduit les formules et par les lois Juliae qui ont supprimé les Actions de la loi. Alors Aulu-Gelle peut écrire que les vades, les subvades, le talion, les vingt cinq as ont disparu à la suite de cette loi qui a permis au magistrat de créer l'action d'injures estimatoire et le vadimonium moderne où c'est le défendeur qui s'engage sur une stipulation ordinaire, bien que les Actions de la loi n'aient été définitivement abolies que depuis les lois Juliae. Au contraire Aulu-Gelle n'aurait pas pu citer la loi Aebutia, aurait dû, tant qu'à citer des lois, citer les lois Juliae, si ce n'était pas la loi Aebutia qui avait permis pour la première fois de se passer des vieilles institutions qui furent abolies par les lois Juliae; Gaius lui-même n'eut pas pu représenter la loi Aebutia comme ayant avec les lois Juliae, aboli les Actions de la loi et introduit les formules, si cette loi, qui n'a pas aboli les Actions de la loi, n'avait pas non plus introduit les formules. Ce n'est pas une bonne note pour nos adversaires que leur système soit directement contredit par les seuls textes qui prononcent le nom de la loi dont il s'agit de fixer la date.

1) Aulu-Gelle, 16, 10,8 (p. 114, n. 3). Même en suivant l'interprétation de M. Wlassak, Proceßgesetze, 1, 102-103. 154-155, où AuluGelle dit, dans ses deux propositions, d'une part que les vades ont disparu (evanuerunt) et, d'autre part que ces vieilleries (illa antiquitas) des XII tables sont sorties de la pratique (consopita) depuis la loi Aebutia sauf dans les affaires centumvirales, la seconde proposition, en parlant de ces vieilleries, se réfère nécessairement aux institutions visées par la première et en rattache l'abandon à la loi Aebutia. 2) Gaius 4, 30 (p. 114, n. 4). 
Nos contradicteurs ne rendent pas mieux compte de la différence si accusée qui se manifeste dans la physionomie générale du droit avant et après le moment où nous plaçons Ia loi Aebutia.

Je ne veux pas répéter les observations que jaai présentées dans mon premier article pour montrer combien cette loi populaire, destinée à réformer la procédure civile par l'introduction de l'écriture, et dont l'histoire politique ne nous rapporte pas le vote, s'enchâsse harmonieusement entre l'an 605 et l'an 628 , ̀̀ une époque pour laquelle nous n'arons plus Tite-Live ni Polybe, dans la période d'aspirations démocratiques qui s'étend de la chute de Carthage à l'échec des Gracques, entre l'an 605 et l'an 673 entre lesquels l'organisation des quaestiones perpetuae réalisa pareillement la réforme de la procédure criminelle, vers le temps qui voit également se développer l'emploi de l'écriture dans les comices où la substitution du vote écrit au vote oral fut essentiellement accomplie entre l'an 615 et l'an 623. Mais je voudrais rappeler, en insistant sur quelques points nouveaux, le témoignage d'ensemble que j'ai signalé comme fourni par la comparaison des temps antérieurs et postérieurs au moment où je place la loi.

J'ai fait là cette comparaison pour les citations que nous avons des jurisconsultes et pour les débris de plaidoiries d'avocats qui nous ont été transmis en prenant comme exemples des deux périodes, pour les jurisconsultes l'auteur du début du VII ${ }^{\mathrm{e}}$ siècle $\mathrm{M}^{\prime}$. Manilius et celui du milieu du VII e P. Rutilius Rufus, pour les orateurs judiciaires, L. Licinius Crassus, né en 614 , mort en 663 , et $M$. Porcius Caton le censeur, né en 520, mort en 605. Quoique nous ayons un peu plus de citations de Manilius que de Rutilius, beaucoup plus de fragments des plaidoyers de Caton que de ceux de Crassus, l'épreure a, dans les deux cas, donné le même résultat: pas une trace de la procédure nouvelle ni dans les citations de Manilius, ni dans les plaidoiries de Caton; des vestiges patents et multiples dans les eitations de Rutilius et dans les plaidoyers de Crassus. On pourrait élargir l'expérience sans en changer le résultat en la faisant porter sur tout le cercle des actions de bonne foi, des actions pré- 
toriennes et des moyens prétoriens; on en dresserait une sorte de statistique avant et après le moment où nous plaçons la loi et, pour rendre la démonstration plus concluante, on $\mathrm{y}$ inscrirait dans la première période non pas les cas avérés, mais ceux qui ont été allégués par des auteurs quelconques: on verrait se manifester entre les deux périodes une diversité si flagrante, une disproportion si écrasante qu'il serait impossible de ne pas y reconnaître l'effet d'une cause positive.

Ici je voudrais signaler deux autres points de vue, l'un relatif encore à la procédure, l'autre en apparence indépendant, desquels l'influence de la loi nouvelle se discerne avec une netteté singulière: celui de la compétence des récupérateurs et celui de l'activité légiférante des assemblées populaires.

Les récupérateurs sont mentionnés, et même assez fréquemment, dans les sources anciennes. Ils le sont par la relation du traité avec les Latins de l'an 261 de la chronologie traditionnelle ${ }^{1}$ ), par des textes de Plaute avant $570^{2}$ ), par Tite-Live vers l'an $583^{3}$ ) et par le sénatus-consulte de Thisbé en l'an 584.4) Mais c'est toujours pour des procès où figurent des pérégrins, jamais entre citoyens. Au contraire ils apparaissent dans les procès entre citoyens aussitôt après la date à laquelle nous plaçons la loi Aebutia: non-seulement dans le cours du VII ${ }^{e}$ siècle pour l'action damni vi dati

1) Denys, 6, 95, dont le témoignage a ici beaucoup plus de valeur qu'il n'en a d'ordinaire pour les temps anciens parce que le traité était encore sous les yeux des Romains au temps de Cicéron (Pro Balbo, 23, 53). V. Organisation judiciaire, 1, pp. 101-102 et les renvois. - ${ }^{2}$ ) Plaute, Rudens, $5,1,1-3$ (procès entre pérégrins). Bacchides, 2, 3, 36-38 (procès entre pérégrin et citoyen). - ${ }^{3}$ ) Tite-Live, 43, 2. - 4) Bruns, Fontes ${ }^{6}$, p. 166 (II, 6). Foucart, Mémoires de l'Acad. des Inscr., 37, 2, 1906, pp. 312-313; cf. pp. 342-343. Les jurés multiples que le sénat vise à deux reprises au pluriel, en répondant aux T'hisbeens que, s'ils veulent demander des juges au sujet de leurs contestations avec Gnaeus Pandosinus, on leur en donnera, sont nécessairement des récupérateurs, si on remarque que l'on n'est ni dans l'un ni dans l'autre des deux cas (bornage et évaluation des rindiciae) où les arbitres sont au nombre de trois et que l'affaire ne rentre pas non plus dans la compétence des collèges des centumvirs et des décemvirs litibus indicandis, dont l'existence est au reste fort douteuse en l'an 584. 
d'après le plaidoyer de Cicéron, Pro Tullio, prononcé entre 681 et $683^{1}$ ), avant Sulla, pour l'action d'injures ${ }^{2}$ ), mais dès

1) Le plaidoyer de Cicéron, Pro Tullio, montre les récupérateurs statuant entre citoyens sur l'action prétorienne damni vi dati introduite en 678, pour les procès où figurent des étrangers, par l'édit du préteur pérégrin Lucullus (cf. Manuel, p. 414, n. 2, trad. allemande, p. 452, n. 3). Or ce plaidoyer, qui est donc postérieur à l'an 678 et qui, d'autre part, appartient sans doute à la première partie de la vie de Cicéron, se place entre l'an 681 et l'an 683; car le procès a été organisé par un préteur nommé Metellus; or jusqu'à Q. Caecilius Metellus Pius Scipio qui, ayant été $t r . p l$. en 694 et cos. en 702, a été préteur au plus tôt en 696 et au plus tard en 699 (Münzer dans Pauly -Wissowa, v. Caecilius, no 99), il ne peut, parmi les Metelli de l'époque, avoir été organisé que par Q. Caecilius Metellus Creticus, qui fut candidat à la préture en 679 et consul en 685 et par conséquent préteur en 680, 681 ou 682 (Münzer, no 87), ou par L. Caecilius Metellus, préteur en 683 (Münzer, $n^{\circ} 74$ ): en effet, des autres Metelli qui ont été préteurs dans l'intervalle, l'un, M. Caecilius Metellus a été pr. repet. en 685 (Münzer, $n^{\circ} 78$ ) et deux autres Q. Caecilius Metellus Celer, pr. 691 (que M. Münzer, n $^{\circ} 86$, dit avec une vieille tradition gratuite avoir été préteur urbain à cause de Cicéron, Pro Sulla, 23,65) et Q. Caecilius Metellus Nepos pr. 694 (Münzer, no 96) ne peuvent avoir été préteurs urbains, la préture urbaine ayant été occupée en 691 par L. Valerius Flaccus (Cicéron, Pro Flacco, 3, 6. 40, 101. In Cat., 3, 2, 5. Salluste, Cat., 45, 1) et en 694 par P. Cornelius Lentulus Spinther (Münzer, dans Pauly-Wissowa, v. Cornelius, no 238, où il ne spécifie pas le caractère de la préture qui résulte de ce que Spinther donna les jeux apollinaires: Mommsen, Staatsrecht, 2, 236, $2=$ tr. fr. 3, p. 271, n. 2). Il faut donc que le Metellus auquel l'affaire a été soumise soit ou L. Caecilius Metellus, qui a été préteur urbain en 683 , ou Q. Caecilius Metellus Creticus qui a éte préteur entre 680 et 682 , wais qui ne peut avoir été préteur urbain qu'en 681 ou 682 , Ia préture urbaine ayant été occupée en 680 par le célèbre Verres. Cela place le procès non pas en 682 ou 683, comme on dit encore parfois (Teuffel, Geschichte der. römischen Literatur; $1^{\text {s }}$, 1890, p.321; mieux Schanz, $1^{2}$, 1898, p. 249, et déjà Drumann, Gesch. Roms, 5, p. 258), mais en 681, 682 ou 683. - 2) L'action d'injures estimatoire déférée aux récupérateurs par l'édit dont parle Labéon chez Aulu-Gelle, 20,1,13, a sans doute été comme la précédente introduite par le préteur pérégrin pour ses justiciables (Hitzig, Iniuria, 1899, pp.60-72). Mais son extension aux citoyens par l'édit du préteur urbain a eu lieu avant Sulla et même assez longtemps avant. C'est évident si, comme nous pensons l'avoir établi, Mélanges Gérardin, pp. 255-282, elle et ses récupérateurs ont été abolis par la loi Cornelia de iniuriis; ce n'est guère plus donteux en dehors de là si l'on considère que le développement qui a remplacé l'idée d'injure 
le lendemain de la réforme, dans un des premiers documents qui l'attestent, dans le passage de la loi latine de Bantia de l'an 636 au plus tard qui est notre premier témoignage direct de l'emploi de la formule entre citoyens. ${ }^{1}$ ) L'opposition est trop nette, appuyée sur des textes trop nombreux pour pouvoir tenir à un hasard de transmission. Mais pourquoi le préteur qui n'avait jamais nommé de récupérateurs entre citoyens jusqu'à la fin du $\mathrm{VI}^{e}$ siècle s'est-il mis à en nommer un peu avant le milieu du VII'? Ne faut-il pas pour cela qu'il y ait eu quelque chose de changé dans ses droits? Et ce changement qui ne s'expliquerait pas autrement, ne s'explique-t-il pas parfaitement si la loi Aebutia n'a été rendue que peu d'années avant la date du VII ${ }^{\mathrm{a}}$ siècle où nous le voyons exercer ce nouveau pouvoir?

La même idée explique comment, malgré l'accroissement continu de l'activité politique et juridique, les comices et l'assemblée de la plèbe paraissent avoir rendu beaucoup moins de lois relatives au droit privé dans la période posté-

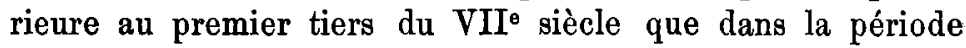
antérieure.

Nous ne connaissons pas loin d'une dizaine de lois relatives au droit privé qu'on attribue, sûrement ou vraisemblablement, au $\mathrm{VI}^{\circ}$ siècle de Rome ou au début du VIIe. Ce sont: la loi Cincia sur les donations de l'an $550^{2}$ ), la loi Plaetoria sur les mineurs de vingt-cinq ans rendue au temps de Plaute, aux environs de l'année $563^{3}$ ); la loi Voconia de $585^{4}$ ); la loi Furia sur les legs qui se place entre la loi Cincia de 550 et la loi Voconia de $585^{5}$ ); enfin la loi Atinia

corporelle exprimée dans cet édit par l'idée moderne d'outrages englobant l'édit de convicio, l'édit de adtemptata pudicitia et l'édit ne quid infamandi causa fiat était déjà accompli avant Sulla (Auct. ad Her., 4, 25, 35. Cf. Mélanges Gérardin, pp. 255-258). C'est prouvé matériellement par l'anecdote du De inventione, 2, 20, 59, Mélanges Gérardin, pp. 268-269, relative à l'injure corporelle faite à un chevalier romain par des hommes armés que rien n'indique être des pérégrins et au sujet de laquelle on se demande s'il faut saisir les récupérateurs ou la justice criminelle.

1) V. les pp. 117, n. 6 , et 153, n. 3. - ${ }^{2}$ ) Cicéron, De senectute, 4, 10. - ${ }^{3}$ ) V. p. 134, n. 2. - ${ }^{4}$ ) Cicéron, De senectute, 5, 14. - ${ }^{5}$ ) V. la p. 134, n. 2 . 
sur l'usucapion discutée comme récente par des jurisconsultes du début du $\mathrm{VI}^{\mathrm{e}}$ siècle ${ }^{1}$ ), toutes les cinq sûres et jusqu'à présent indiscutées ${ }^{2}$ ); ensuite, au moins dans la doctrine qui me semble la meilleure et que je crois la plus répandue, les deux lois Appuleia et Furia sur le cautionnement, dont la plus ancienne est postérieure à la création de la province de Sicile en 513 et la plus récente antérieure à la loi Vallia, cette loi Vallia que nous croyons de la fin du VI siècle ou du début du VII ${ }^{e}$, et enfin la loi Crepereia qui parait postérieure à la réduction de la valeur de l'as opérée en 537 et tout au moins antérieure à la nouvelle réduction faite en 665, qu'il y a même des raisons de juger sensiblement plus ancienne. ${ }^{3}$ ) Exactement neuf lois dont quatre

1) Aulu-Gelle, 17, 7. - $\left.{ }^{2}\right)$ M. Mitteis, Privatrecht, p. 52, n. 30, a manifesté l'intention de contester pour la loi Furia testamentaria le raisonnement de Bruns; mais il ne l'a pas encore fait. La radiation d'une loi sur cinq ne changerait d'ailleurs pas grandement la force de notre argumentation. $-{ }^{3}$ ) La doctrine qui place les deux lois Appuleia et Furia dans le VIe siècle parait toujours la plus répandue (v. en dernier lieu Levy, Sponsio fidepromissio fideiussio, 1907, p. 63 et les auteurs cités), quoiqu'elle ait été combattue avec persistance par $M$. Appleton qui veut rattacher la loi Appuleia à Saturninus tr. pl. en 651 et 654 et la loi Furia à son contemporain Furius (articles cités p. 135, n. 2 et déjà $R$. Historique de droit, 1876, pp. 541-581) et que cela conduirait par ex. à faire descendre encore plus bas, presqu'au temps de Cicéron la loi Vallia dont nous avons déjà parlé p. 124. Pour la loi Crepereia qui, d'après Gaius 4, 95, fixe à 125 sesterces le montant de la sponsio par laquelle sont saisis les centumvirs, le langage de Gaius nous parait devoir s'entendre de la sponsio sur laquelle portera le sacramentum et non pas du sacramentum lui-même. Mais nous jugeons pourtant impossible de ne pas tenir compte de l'observation lumineuse de Mommsen, Staatsrecht, 2, $231=$ trad. fr. 3, 265, 2, selon laquelle la somme de 125 sesterces correspond, à l'époque où l'as vaut 4 sesterces, exactement au montant du sacramentum le plus élevé, du sacramentum de 500 as de Gaius, 4,14. La loi nous parait donc avoir été faite pour le sacramentum, duquel ce taux peut ensuite avoir été transporté à la sponsio préjudicielle récente de mille façons, par ex. dans la disposition qui a permis d'intenter les vindicationes soumises aux centumvirs par une sponsio donnant lieu à une legis actio sacramenti in personam au lieu de le faire correctement par une legis actio sacramenti in rem. Mais alors la loi doit, comme a vu Mommsen, être nécessairement postérieure à la réduction de l'as à 4 sesterces opérée en 537, et pour la même raison, elle doit, je crois être antérieure à 
plus ou moins discutées, mais dont tout au moins cinq sûres.

$\mathrm{Au}$ contraire, une fois les premières années du VII ${ }^{e}$ siècle passées, on ne trouve plus, même en attribuant au dictateur Sulla toutes les lois Corneliae et en comptant la loi Cicereia, que je pense de cette période, mais que d'autres mettent au VIe siècle, que trois ou quatre lois de droit privé, elles-mêmes en général provoquées par le contrecoup de réformes pénales ou de mesures politiques: la loi Cicereia ${ }^{1}$ ), la loi Cornelia sur le cautionnement ${ }^{2}$ ), la loi Cornelia sur l'hérédité du citoyen mort chez l'ennemi ${ }^{3}$ ) et la loi Plautia sur l'usucapion des choses enlevées par violence, qui est une partie de la loi Plautia de vi rendue entre l'époque de Sulla et la conjuration de Catilina. ${ }^{4}$ ) Pour en trouver une cinquième, il

la nouvelle réduction de l'as opérée en 665 par la loi Papiria (Pline, 33,46 ) après laquelle le taux du sacramentum eut été moindre de moitié. Il me semble en outre aujourd'hui qu'il existe un autre élément qui enferme la loi plus strictement dans cette période. D'après le rapport dans lequel Gaius la met avec les procès déférés aux centumvirs, il y a tout lieu de croire que, même si elle n'a pas été, comme pense M. Mommsen, la loi qui les a créés, elle a tout au moins été faite au moment de leur création pour fixer le taux du sacramentum sur lequel on plaiderait devant eux. Or le tribunal des centumvirs existe déjà à une époque voisine de l'an 618 , vers laquelle on trouve, suivant l'observation de M. Olivier Martin, Tribunal des centumvirs, 1904, p. 5, la mention du plus ancien procès connu déféré aux centumvirs, du procès relatif à la condition de Mancinus le consul de 617, fait sans doute peu après que son extradition avait été refusée par les Numantins en 618 (Cicéron, De orat., 1, 56, 238; cf. 1, 40, 181-182).

1) Gaius, 3, 128. Manuel, 756, $2=$ tr. all. 826, 1. M. Lévy, p. 65, l'attribue au préteur de 581 mentionné par Tite-Live en disant que l'argument tiré de l'identité de nom dont il ne méconnait pas la défectuosité générale acquiert ici plus de valeur à cause de la rareté du gentilice. - ${ }^{2}$ ) Gaius, 3, 124-125. Loi sûrement antérieure à la loi Julia d'Auguste sur la vicesima hereditatis, d'après Gaius, 3, 125, et sûrement postérieure à la loi Cicereia, si on admet qu'elle est postérieure à la création des fidéjusseurs, comme on le pense généralement, mais comme M. Levy, p. 118, le nie. - ${ }^{3}$ ) Paul, Sent., 3, 4a, 8. Cf. Manuel, 191, 3; trad. 213, 2. - 4) Gaius, 2, 45, qui l'accole, au sujet de la prohibition d'usucaper les choses enlevées par violence, à une loi Julia de César ou d'Auguste sous le nom collectif de lex Iulia et Plautia. Elle se place sûrement entre l'an 673, date des lois criminelles de Sulla qu'elle vient compléter, et l'an 691, dans lequel elle 
faudrait descendre jusqu'à l'an 714, date de la loi essentiellement politique proposée par le tribun Falcidius pour assurer le rendement d'un impôt sur les successions testamentaires en garantissant à l'héritier un quart de la succession à l'encontre des légataires. ${ }^{1}$ ) Jusque-là, la seule matériellement sûre est la loi Plautia. En résumé donc, en écartant, d'un côté comme de l'autre, les cas douteux ou contestés, une loi sûre contre cinq. Cependant, l'on soutiendrait malaisément que la vie politique ait été plus active, le progrès juridique plus intense dans la période où l'on trouve cinq lois que dans celle où l'on n'en trouve qu'une. Seulement dans l'intervalle la loi Aebutia a été rendue. Les réformes qui étaient faites auparavant par le vote du peuple sont faites désormais par l'édit du préteur. C'est la loi Aebutia qui explique cette raréfaction brusque des lois relatives au droit privé comme tous les autres symptomes d'un état nouveau qui se rencontrent dans la même période et elle explique tous ces phénomènes concordants au moment précis où elle devait les produire d'après la date que nous lui avons attribuée.

Nous aurions craint de paraitre nous perdre dans le vague, en commençant par ces considérations d'ensemble. Mais, présentées en leur lieu, elles ne fournissent pas une mauvaise confirmation des raisonnements directs et spéciaux par lesquels nous avons obtenu notre date. Puisqu'il s'agit de la solution d'un problème, on pourrait dire que e'est, sur le terrain de l'histoire générale, la preuve de l'opération par laquelle nous avons, sur le terrain des faits juridiques isolés, placé la loi dans le premier tiers du VII siècle de Rome, à une date postérieure à l'an 605 et antérieure à l'an 628 de la chronologie varronienne.

fut invoquée contre Catilina d'après Salluste, Cat. 31, 4, peut-être en l'an 677, comme a pensé Mommsen, Strafrecht, 654, $2=$ tr. fr. 2, 373, 4, à cause de Cicéron, Pro Caelio, 39, 70.

1) Diou, 48,33 . 